\title{
Linx
}

Revue des linguistes de l'université Paris X Nanterre

5 | 1994

La négation

\section{L'évolution des propositions infinitives négatives en français}

\section{Paul Hirschbühler et Marie Labelle}

\section{(2) OpenEdition}

\section{Journals}

Édition électronique

URL : http://journals.openedition.org/linx/1194

DOI : 10.4000/linx.1194

ISSN : 2118-9692

\section{Éditeur}

Presses universitaires de Paris Nanterre

\section{Édition imprimée}

Date de publication : 1 juin 1994

Pagination : 59-90

ISSN : 0246-8743

\section{Référence électronique}

Paul Hirschbühler et Marie Labelle, "L'évolution des propositions infinitives négatives en français », Linx [En ligne], 5 | 1994, mis en ligne le 18 juillet 2012, consulté le 20 avril 2019. URL : http:// journals.openedition.org/linx/1194; DOI : 10.4000/linx.1194

Ce document a été généré automatiquement le 20 avril 2019.

Département de Sciences du langage, Université Paris Ouest 


\title{
L'évolution des propositions infinitives négatives en français
}

\author{
Paul Hirschbühler et Marie Labelle
}

\section{Introduction}

1 Le présent article s'inscrit dans une étude plus générale de l'évolution des aspects de la grammaire du français responsables de la position relative du verbe infinitif et de certains éléments qui gravitent dans son orbite, à savoir, la négation, certains adverbes et les pronoms clitiques. Nous examinons ici la position du verbe infinitif par rapport au forclusif pas. L'analyse proposée a été en partie stimulée par certains détails de la description et de l'analyse de la syntaxe des propositions infinitivales négatives que l'on trouve dans l'important article de Jean-Yves Pollock (1989), Verb Movement, Universal Grammar and the Structure of IP. Nous résumerons et discuterons brièvement les aspects pertinents de l'analyse de Pollock concernant la position relative du verbe, de la négation et de certains adverbes dans les propositions à temps conjugué avant d'en venir à l'examen et à la discussion des données historiques, et à notre propre analyse.

\section{L'analyse de Pollock}

2 L'article de Pollock (1989) représente une des contributions les plus marquantes de la dernière décennie à certains aspects du programme de recherche de la théorie des paramètres en grammaire générative, et son importance est confirmée par les nombreuses études qu'elle a suscitées sur la syntaxe des catégories fonctionnelles de diverses langues. Son analyse s'est révélée une source d'inspiration à deux égards au moins. D'une part, l'idée de séparer certaines catégories fonctionnelles complexes, comme Infl, qui regroupe les marques de temps (T) et de personne-nombre caractéristiques de l'accord du verbe avec le sujet (Agr), en catégories fonctionnelles distinctes ( $\mathrm{T}$, Agr, et d'autres, comme la modalité dans les langues balkaniques - cf. Rivero 1988, 1990) a favorisé un renouvellement de l'étude de la syntaxe des éléments 
traditionnellement rattachés à la flexion verbale: temps, aspect, négation, clitiques. D'autre part, l'analyse de Pollock représente un effort systématique pour faire découler de principes de la grammaire universelle l'application ou la non-application de certains processus transformationnels. Dans chacun de ces cas, la démarche générale suivie importe plus que les solutions particulières, les propositions d'analyse dans ce domaine se succédant rapidement. Les données que nous discuterons nous amèneront également à reconsidérer divers aspects des propositions de Pollock ou d'autres linguistes qui se sont inspirés de son travail dans leur discussion de certaines des données historiques discutées ici.

3 Pollock suggère le schéma (la) comme représentation typique, éventuellement simplifiée, de la structure de base (désormais, la D-structure) des phrases négatives du français, que le verbe soit à temps conjugué ou à l'infinitif. De bas en haut, on note :1) VP, la projection maximale du verbe ; 2) un syntagme AgrP, dont la tête Agr(eement) contient les traits d'accord du verbe avec le sujet ; 3) NegP, un syntagme négatif représentant la négation de phrase, dont la tête est le morphème ne; la réalisation typique éventuelle du spécifieur de cette catégorie est pas ; 4) La catégorie fonctionnelle maximale la plus haute, si l'on s'arrête avant le système du complémenteur, est une projection de $\mathrm{T}$ (emps), le site de la flexion temporelle. T est spécifié [+ fini] dans le cas des verbes à temps conjugué et [-fini] dans le cas des verbes à l'infinitif (cf. Pollock, p. 372). La structure donnée en (la) ne représente que le cas des temps simples, avec une seule occurrence de VP; dans le cas de temps composés, l'auxiliaire est simplement introduit sous $\mathrm{V}$ et sélectionne lui-même un autre VP. L'arbre (1b), où AgrP et TP sont intervertis, est une alternative plus commune à la représentation originale de Pollock.

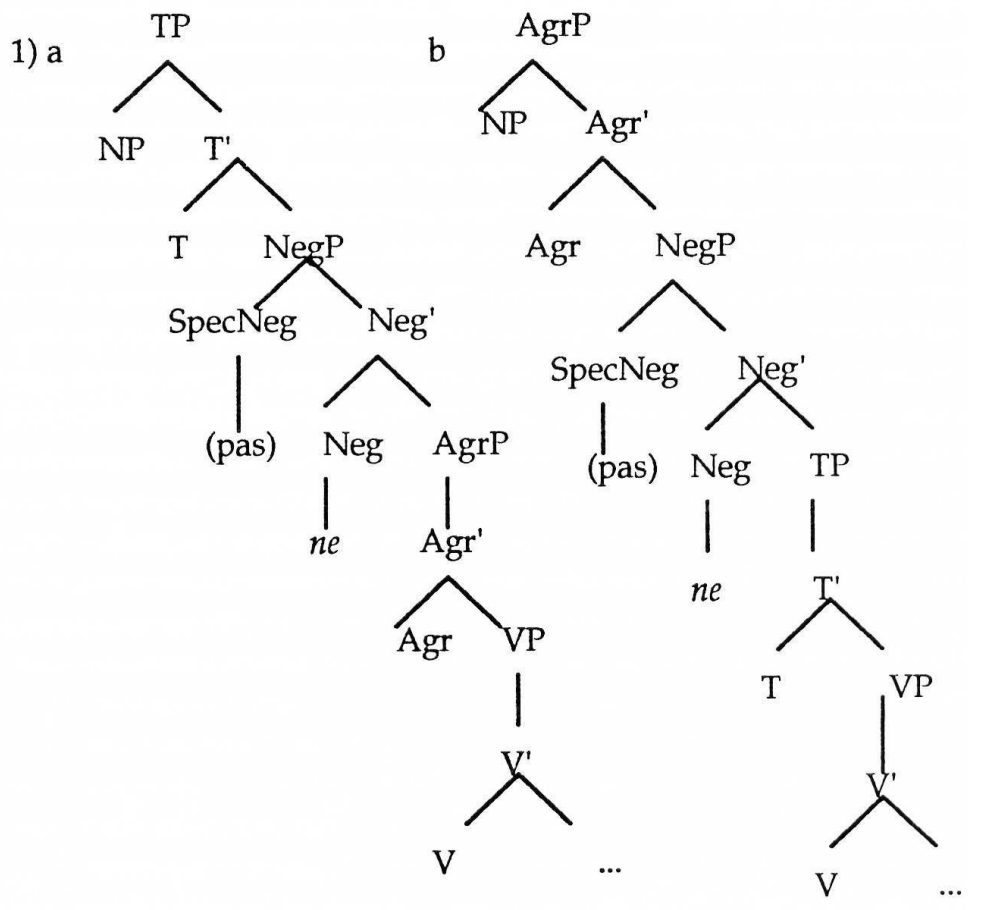

(2) a Pierre n'aimepasles exposés trop longs. 
b Pierre n'apas raccourci notre exposé.

Dans l'hypothèse d'une D-structure du type de (1a), il convient de développer une analyse motivée de la manière dont se réalise l'amalgame des divers affixes flexionnels avec la racine verbale, ainsi que des opérations requises pour rendre compte des aspects de l'ordre des mots qui distinguent l'ordre de surface de l'ordre proposé comme sous-jacent. ${ }^{1}$ Edmonds (1978) a déjà montré que dans la perspective d'une analyse où la flexion verbale est représentée indépendamment du verbe, l'amalgame du verbe et de la flexion doit être $\mathrm{vu}$, dans le cas des verbes conjugués du français, comme le résultat d'un processus déplaçant le verbe vers la flexion. Cette analyse est reprise par Pollock, sous la forme d'un déplacement initial de $\mathrm{V}$ à Agr, auquel $\mathrm{V}$ s'adjoint, suivi du déplacement et de l'adjonction de Agr et du matériel qui s'est adjoint à lui (c.à.d. de [Agr V+Agr]) à $T$, ces diverses adjonctions correspondant à des incorporations successives de la tête déplacée (simple ou complexe) à la tête hôte. Dans le courant de recherches étroitement associé au programme chomskyen, l'hypothèse générale d'un déplacement du verbe hors de VP vers des têtes fonctionnelles supérieures est aujourd'hui généralement adoptée pour la syntaxe du verbe conjugué en français, modulo une D-structure alternative, où, au minimum, T et Agr son intervertis, comme en (1b) (cf. Belletti 1990 et Chomsky 1991), une proposition à laquelle Pollock lui-même (séminaire UQAM, été 1991) n'est pas resté insensible.

6 L'ordre relatif de pas et de ne est analysé par Pollock, et par d'autres à sa suite, comme le résultat du déplacement de ne au niveau de la catégorie fonctionnelle la plus élevée dans un schéma du type de (1), c.à.d. T en (la) (Agr en (1b)). Le déplacement de ne est ainsi conçu parallèlement au déplacement postulé pour les pronoms clitiques objets.

7 Pour ce qui est des propositions infinitivales, Pollock propose que, dans le cas des verbes que l'on appelera informellement verbes pleins, c'est-à-dire les verbes autres que avoir, être et les modaux pouvoir, vouloir et devoir, le verbe (la racine verbale) ne se déplace pas jusqu'à la tête fonctionnelle la plus élevée dans l'arbre, l'absence de mouvement rendant compte du fait qu'en français contemporain, le verbe à l'infinitif suit pas :

\begin{tabular}{|l|l|}
\hline (3) & a J'espère nepasvousretenir au-delà de l'heure prévue. \\
\hline & b *'espère nevousretenirpas au-delà de l'heure prévue. \\
\hline
\end{tabular}

8 Afin de rendre compte d'alternances du type de celle notée en (4a-b), il est proposé que le verbe à l'infinitif est sujet à un déplacement facultatif de sa position de base à une tête fonctionnelle immédiatement supérieure à VP, c.à.d. Agr dans l'hypothèse (1a).

\begin{tabular}{|l|l|}
\hline (4) & a Jean avait honte detoujoursdéranger son voisin. \\
\hline & b Jean avait honte dedérangertoujours son voisin. \\
\hline
\end{tabular}

9 Ceci requiert évidemment l'hypothèse additionnelle qu'un adverbe du type de ceux apparaissant aussi bien à la gauche qu'à la droite immédiate du verbe à l'infinitif occupe 
une position fixe d'adjonction à VP. Dans le cas de (4a) le verbe apparait dans sa position de base. Dans le cas de (4b), il s'est déplacé à Agr. L'absence de montée de V, ou plus exactement de $\left[_{\mathrm{Agr}} \mathrm{V}+\mathrm{Agr}\right]$, à $\mathrm{T}$ est expliquée par l'hypothèse que $\mathrm{T}[$-fini] est opaque à la transmission des rôles thêta par le verbe à ses arguments éventuels (Pollock, 1989 :386). La catégorie Agr est considérée comme suffisamment riche en français pour être transparente à la transmission des rôles thêta - le contraire est suggéré pour l'anglais bien qu'elle n'ait pas de réalisation morphologique dans le cas des infinitives (Pollock, 1989 :385-386). Lorsqu'il n'y a pas mouvement de V (ou V+tête fonctionnelle) vers une tête fonctionnelle supérieure, c'est celle-ci, qu'elle ait une réalisation phonétique ou non, qui descend s'adjoindre à la tête de la catégorie fonctionnelle immédiatement inférieure dans la structure.

10 En ce qui concerne les infinitives ayant pour tête être,avoir et un verbe modal, l'analyse de Pollock peut se résumer de la manière suivante.

11 1. Avoir et être (appelés " auxiliaires ", même dans leur emploi comme copules) peuvent, mais ne doivent pas, monter à une catégorie fonctionnelle à gauche de pas et d'adverbes comme souvent. Selon l'auteur, les exemples mettant en jeu ces verbes sont généralement considérés comme quelques peu littéraires et recherchés, mais indiscutablement bons ("perfectly fine ») (Pollock, p. 373-374). Ce déplacement est attribué au fait que ces verbes, dans leurs divers emplois, n'ont pas de grille thématique, donc pas de rôles thêta à transmettre (Pollock, p. 386-389).

\begin{tabular}{|l|l|}
\hline (5) & a Etre ou ne pas être, telle est la question \\
\hline \hline & b Etre ou n'être pas, telle est la question \\
\hline (6) & a Ne pas avoir de voiture en banlieue rend la vie difficile \\
\hline & b N'avoir pas de voiture en banlieue rend la vie difficile. \\
\hline
\end{tabular}

12 2. Les modaux devoir, pouvoir et vouloir sont décrits comme pouvant monter à $\mathrm{T}$, le résultat ayant une résonance très littéraire signalée par le point d'interrogation en $(7 b),(8 b)$ et (9b), point d'interrogation qui n'est pas ici le signe d'une acceptabilité douteuse (Pollock, p. 375).

\begin{tabular}{|l|l|}
\hline (7) & a Je pensais ne pas pouvoir dormir dans cette chambre. \\
\hline \hline & b ?Je pensais ne pouvoir pas dormir dans cette chambre. \\
\hline$(8)$ & a Il avait estimé ne pas devoir donner suite à ma demande. \\
\hline & b ?Il avait estimé ne devoir pas donner suite à ma demande. \\
\hline (9) & a Il avait dit ne pas vouloir donner suite à ma demande. \\
\hline & b ?Il avait dit ne vouloir pas donner suite à ma demande. \\
\hline
\end{tabular}


13 Afin de rendre compte des jugements rapportés, il est proposé que le sujet et le complément propositionnel de ces verbes ne recevraient pas de rôles thêta argumentaux ordinaires, mais des rôles thêta d'une nature différente (des rôles thêta adjoints) qui ne seraient pas sensibles à l'effet d'opacité de T[-fini] (Pollock, p. 389-391, en particulier la note 28$)^{2}$

Comme nous le verrons ci-dessous, l'importance générale et le bien fondé de la tripartition entre verbes pleins, modaux et auxiliaires comme facteurs importants pour la description et l'analyse est appuyée par les faits typiques de certaines périodes du français et par certains aspects de l'évolution. Cependant, les jugements rapportés par Pollock ne nous paraissent pas refléter véritablement la situation du français ordinaire contemporain dans le cas des auxiliaires et des modaux, qu'il s'agisse de la langue parlée ou écrite. Dans le cas des modaux, les jugements des locuteurs tendent à être négatifs lorsque l'infinitif est encadré par ne et pas. Pour ce qui est des verbes avoir et être, les jugements semblent devoir être raffinés et nuancés en distinguant l'emploi d'auxiliaire de celui de copule.

\section{L'évolution des infinitivales négatives}

15 Résumons d'abord brièvement les grandes lignes de l'évolution des subordonnées infinitivales négatives en français. Les premières subordonnées infinitivales négatives sont généralement introduites par non. Ne apparaît dans ce type de subordonnée vers la fin du XIVe siècle et alterne avec non durant le XV ${ }^{\mathrm{e}}$ siècle (Pearce 1990b, 1991) ${ }^{3}$. Seules les infinitives en ne retiendront notre attention ici. Parmi celles-ci, nous ne considérons que celles où le forclusif est de type pas, point, mie (pour alléger la présentation, ces forclusifs seront désignés par pas), à l'exclusion d'éléments comme jamais, rien, plus dont la distribution n'est pas toujours identique. Comme nous le verrons plus en détail cidessous, après la période des XVe-XVIe siècle où, dans le cas d'une négation de type ne...pas, les verbes des différentes classes (pleins, modaux, auxiliaires) devaient normalement précéder $\mathrm{pas}^{4}$, un ordre alternatif, avec $\mathrm{V}$ à droite de pas, se développe, d'abord pour les verbes pleins, puis pour les modaux, et enfin pour être et avoir. Dans le cas des verbes pleins tout d'abord, et plus récemment des modaux, cette nouvelle construction est devenue la règle.

16 Les exemples de (10) illustrent la construction initiale, (10a) étant la première attestation signalée de ne...pas en subordonnée infinitive. Les trois derniers exemples illustrent le phénomène dans le dernier quart du XVIIe siècle pour les trois classes de verbes.

\begin{tabular}{|l|l|}
\hline 10) & $\begin{array}{l}\text { a. ...pour ce, mes, chieres filles, est-il bon de ne se haster point ... (La Tour Landry, 1372, } \\
\text { p. 102). }\end{array}$ \\
\hline & $\begin{array}{l}\text { b. Lors la dame, qui voit bien que elle a congié, fait semblant que elle amast mieux n'y } \\
\text { aller point et dit... (XV joies, c. } 1400,2,15) .\end{array}$ \\
\hline \hline & $\begin{array}{l}\text { c. Ce qui est difficile, c'est de ne s'abandonner pas au plaisir de les suivre. (Mme de } \\
\text { Lafayette, Clèves, } 1678, \text { p. } 94)\end{array}$ \\
\hline & \begin{tabular}{l} 
d. Il croyait même n'en devoir pas trouver ... (Mme de Lafayette, Clèves, 1678, p. 177) \\
\hline
\end{tabular}
\end{tabular}


e. ... des reproches de ne l'avoir pas vu. (Mme de Lafayette, Clèves, 1678, p. 195).

17 La construction moderne, avec le verbe infinitif à la droite du forclusif, est d'abord attestée dans une construction où les pronoms clitiques éventuels se trouvent à la gauche plutôt qu'à la droite de pas. Les exemples en (11) illustrent cette étape. L'exemple de Vigneulles représente la première attestation que nous connaissions de cette construction.

\begin{tabular}{|c|c|}
\hline \multirow[t]{2}{*}{ (11) } & $\begin{array}{l}\text { a. ... car par ainsi pourrés estre quicte de n'en point donner. (Vigneulles, CNN, 1505-1515, } \\
52 / 115)\end{array}$ \\
\hline & $\begin{array}{l}\text { b. Et ai-je lieu de n'en pas espérer un succès aussi favorable? (Cyrano de Bergerac, } \\
\text { Voyage, 1655, p. 31). }\end{array}$ \\
\hline \multicolumn{2}{|r|}{$\begin{array}{l}\text { c. ...afin de ne vous pas trouver mêlée dans des aventures de galanterie. (Mme de la } \\
\text { Fayette, Clèves } 1678, \text { p. } 65 \text { ) }\end{array}$} \\
\hline \multicolumn{2}{|r|}{ d. ...alarmé de ne le pas voir (Cazotte, Diable A., 1772, p. 122) } \\
\hline & $\begin{array}{l}\text { e. Songez que je puis ne le pas vouloir longtemps (Crébillon, Les égarements, 1736-1738, } \\
\text { p. 202) }\end{array}$ \\
\hline & $\begin{array}{l}\text { f. L'élévation cependant était assez grande pour le beaucoup blesser (Cyrano de } \\
\text { Bergerac, Voyage, 1655, p. 42) }\end{array}$ \\
\hline & $\begin{array}{l}\text { g.... si on eût eu autant de soin de les bien conduire dans le progrès de leur âge... } \\
\text { (Bossuet, Discours, } 1682, \text { p. 381) }\end{array}$ \\
\hline
\end{tabular}

18 Cette construction est rare au XVIe siècle mais courante au XVIIe siècle (11b-c). L'exemple de Mme de la Fayette illustre l'alternance entre verbe plein à gauche ou à droite de pas chez le même auteur (voir 10c); les exemples (11d-e), montrent que l'indépendance du clitique par rapport au verbe infinitif se poursuit au XVIIIe siècle ${ }^{5}$, et les deux derniers visent à montrer que, dans l'étude de l'évolution du placement du clitique, la position de certains adverbes non négatifs est également importante, puisqu'ils peuvent intervenir entre le pronom et l'infinitif, ce qui n'est généralement plus le cas aujourd'hui.

19 Finalement, la construction actuelle, avec le verbe infinitif et les pronoms clitiques éventuels à la droite du forclusif, est illustrée en (12) pour diverses époques où la présence du verbe à la gauche du forclusif est également attestée avec le même type de verbe. L'existence d'une variation est potentiellement importante pour le choix entre diverses analyses.

(12) a.... ils étaient délibérés de ne point rendre le prisonnier... (Vigneulles, Journal, fin XVedébut XVIe, p. 92) 


\begin{tabular}{|l|l|}
\hline & $\begin{array}{l}\text { b. ..., parce que la république aime mieux ne point avoir d'enfants, que d'en avoir de } \\
\text { semblables à eux. (Cyrano, 1655, p. 85) }\end{array}$ \\
\hline & c. ..., nous fûmes bien malheureux de ne pas t'emmener ... (Cyrano, 1655, p. 127) \\
\hline
\end{tabular}

20 La première question soulevée par ce genre de données est évidemment descriptive et concerne l'établissement précis des faits d'évolution. La figure 1 permet de se faire une idée générale, pour chaque classe de verbe, du passage progressif de l'ordre ne $V$ pas à ne pas $\mathrm{V}$, abstraction faite de la position des clitiques objets et d'adverbes comme bien. Les données qui sous-tendent la figure 1 sont données dans la Table $1^{6}$. Elles indiquent pour chaque type de verbe et chaque période 1) le nombre total d'occurrences de ce type ne (...) V pas ; 2) de type ne (...) pas (...) V ; 3) la fréquence de la construction moderne, abstraction faite de la position des clitiques éventuels, basée sur les deux lignes précédentes ; 4) la fréquence de la construction moderne basée sur la fréquence notée chez chaque auteur.

21 Figure 1

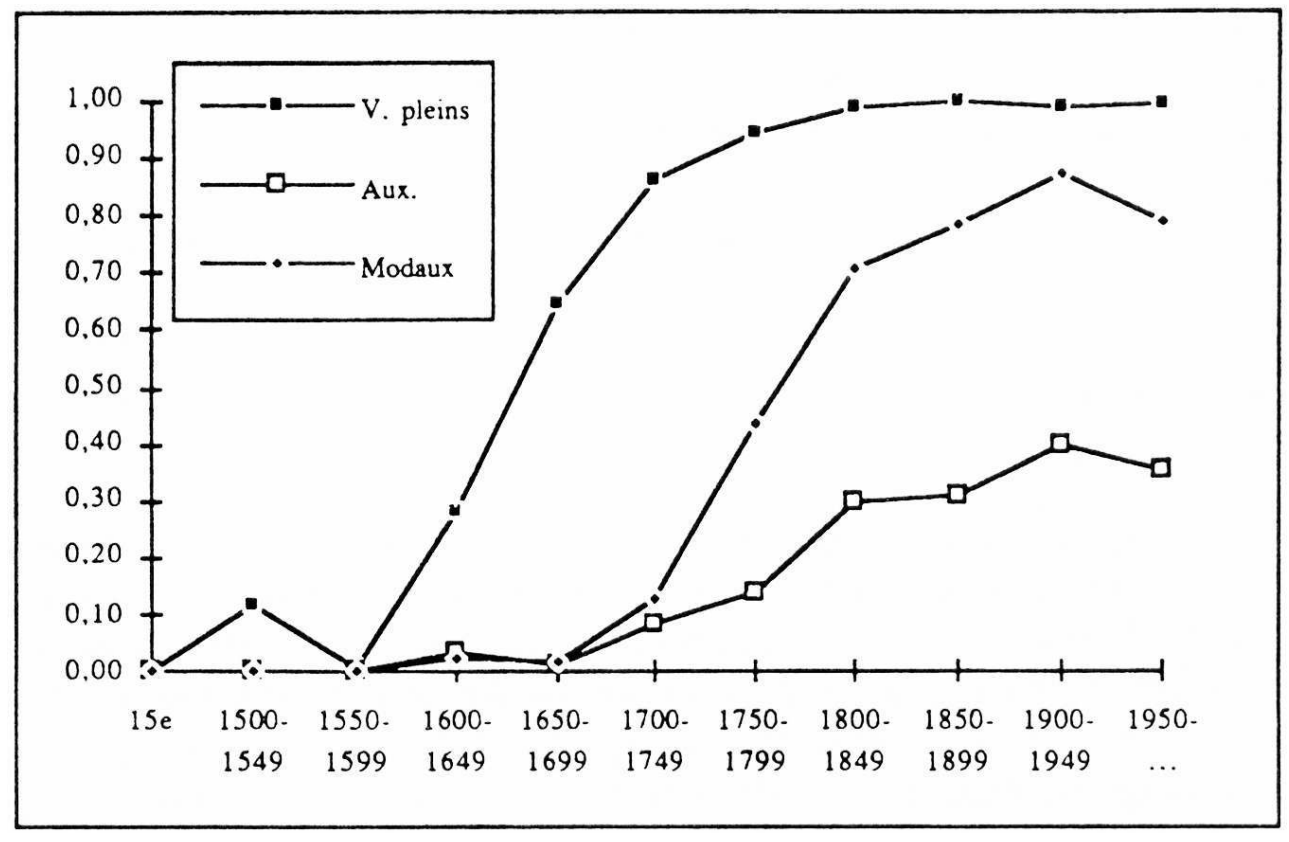

Table 1. Position du verbe infinitif relativement à paset point

\begin{tabular}{|l|l|l|l|l|l|l|l|l|l|l|l|}
\hline & $\begin{array}{l}15 \mathrm{e} \\
\mathrm{s}\end{array}$ & 1500 & 1550 & 1600 & 1650 & 1700 & 1750 & 1800 & 1850 & 1900 & 1950 \\
\hline & & -1549 & -1599 & -1649 & -1699 & -1749 & -1799 & -1849 & -1899 & -1949 & - \\
\hline V. Lexical & & & & & & & & & & & \\
\hline ne...V p & 1 & 65 & 111 & 774 & 400 & 79 & 16 & 5 & 4 & 25 & 4 \\
\hline ne...p...V & 0 & 9 & 2 & 379 & 608 & 436 & 784 & 1346 & 1138 & 1029 & 484 \\
\hline
\end{tabular}




\begin{tabular}{|c|c|c|c|c|c|c|c|c|c|c|c|}
\hline Fréq. ne...p...V & 0.00 & 0.12 & 0.02 & 0.33 & 0.60 & 0.85 & 0.98 & 1.00 & 1.00 & 0.98 & 0.99 \\
\hline $\begin{array}{l}\text { Moyenne fréq./ } \\
\text { auteur }\end{array}$ & 0.00 & 0.12 & 0.01 & 0.28 & 0.65 & 0.86 & 0.94 & 0.99 & 1.00 & 0.99 & 0.99 \\
\hline \multicolumn{12}{|l|}{ V. auxiliaire } \\
\hline ne... V p & 0 & 13 & 38 & 426 & 274 & 142 & 286 & 303 & 263 & 210 & 100 \\
\hline ne...p...V & 0 & 0 & 0 & 6 & 9 & 24 & 37 & 138 & 108 & 138 & 42 \\
\hline Fréq. ne...p...V & - & 0 & 0.00 & 0.01 & 0.03 & 0.14 & 0.11 & 0.31 & 0.29 & 0.40 & 0.30 \\
\hline $\begin{array}{l}\text { Moyenne fréq./ } \\
\text { auteur }\end{array}$ & 0.00 & 0.00 & 0.00 & 0.04 & 0.01 & 0.08 & 0.14 & 0.30 & 0.31 & 0.40 & 0.35 \\
\hline \multicolumn{12}{|l|}{ V. modal } \\
\hline ne...V p & 0 & 2 & 8 & 85 & 81 & 94 & 86 & 40 & 26 & 41 & 4 \\
\hline ne...p...V & 0 & 0 & 0 & 5 & 3 & 17 & 49 & 78 & 120 & 253 & 21 \\
\hline Fréq. ne...p...V & - & 0.00 & 0.00 & 0.06 & 0.04 & 0.15 & 0.36 & 0.66 & 0.82 & 0.86 & 0.84 \\
\hline $\begin{array}{l}\text { Moyenne fréq./ } \\
\text { auteur }\end{array}$ & - & 0.00 & 0.00 & 0.02 & 0.02 & 0.13 & 0.44 & 0.71 & 0.78 & 0.87 & 0.79 \\
\hline
\end{tabular}

Il est clair que le français est passé d'un système où le verbe à l'infinitif, quel qu'il soit, se place devant pas à un système où il apparaît, à peu de choses près, toujours après lui. On note aussi qu'alors que $80 \%$ des verbes pleins se placent à droite de pas dès la première moitié du XVIIIe siècle, il faut attendre la fin du XIXe siècle pour qu'un pourcentage semblable de modaux se retrouve dans cette position. ${ }^{7}$ Si l'on se fie à l'étude de Engver (1972), on ne trouve plus, sauf exceptions, de modaux à gauche du forclusif dans les ouvrages parus entre 1940 et 1963. A cette même époque, toujours d'après Engver, les auxiliaires se retrouvent encore dans cette position dans près de $65 \%$ des cas, tous auteurs confondus, mais ce pourcentage tombe à $29 \%$ si l'on exclut Montherlant et Marcel Aymé, dont la grammaire se démarque clairement de celle des autres auteurs étudiés ${ }^{8}$. On peut donc estimer qu'en ce qui concerne la disparition de l'ordre ne $V$ pas, il y a un décalage d'un siècle et demi environ entre les verbes pleins et les modaux, alors que la construction ancienne était encore majoritaire avec les auxiliaires jusque vers 1964, date des derniers textes de ARTFL.

Les grandes lignes de l'évolution de la position relative du verbe infinitif et de pas étant cernées, il reste à déterminer la structure et la dérivation des infinitivales négatives à chaque époque. Nous discuterons des analyses antérieures des données historiques, avant de présenter nos propositions d'analyse. Le choix d'une analyse pour la construction typique d'une classe de verbes particulière à une époque donnée oriente ou limite dans une certaine mesure la manière dont on pourra expliquer le passage à la nouvelle construction pour cette même classe ; de la même manière, l'observation éventuelle que deux changements (ou plus) sont contemporains, ou que l'un précède de peu l'autre, peut 
suggérer que les deux sont liés, et ceci peut également nous conduire à analyser telle construction à une époque donnée d'une certaine façon plutôt que d'une autre. Nous verrons que la prise en compte des données d'évolution conduit à préférer certaines analyses à d'autres.

\section{Une analyse en termes de barrières}

Jusqu'ici il n'y a pas eu d'essai d'explication qui tienne compte des différences notées ici pour les trois classes de verbes. Les analyses de Martineau $(1990,1992)$ et de Pearce (1990b, 1991) visant à rendre compte du passage de ne V pas à ne pas V ont essentiellement pris en compte les données mettant en jeu les verbes pleins. Ces analyses font référence, de manière très différente dans chaque cas, à la théorie des barrières de Chomsky (1986) pour expliquer l'évolution des constructions étudiées ici. L'analyse de Pearce (1991), bien que très intéressante en ce qui concerne les différences d'ordres de mots associées au choix de non ou ne dans les infinitives, ne semble pas de nature à permettre une explication au fait que le passage de l'ordre ne $V$ pas à ne pas $V$ se produit à des époques différentes pour les verbes pleins, les modaux, et les auxiliaires. Nous nous limiterons dès lors à une discussion de l'analyse de Martineau, fondée sur l'hypothèse de l'apparition d'une barrière à la montée du verbe. Notre but est de contraster ce type d'analyse avec une approche alternative selon laquelle la position du forclusif lui-même aurait changé.

\subsection{Barrière et appauvrissement de la flexion verbale}

La richesse morphologique de la flexion verbale a été associée, dans le cas de langues comme l'italien, à l'existence d'un certain nombre de propriétés comme la possibilité d'expression nulle pour le sujet, la position postverbale du sujet nominal, l'existence d'infinitifs substantivés, la montée longue du clitique objet et la montée du verbe dans Agr, tant dans les phrases à l'infinitif que dans celles à temps conjugué (cf. notamment, Kayne 1989, Martineau 1988, 1990, Rizzi 1982, Rochette 1988). Dans sa thèse, véritable mine d'informations et d'analyses originales sur la syntaxe des propositions infinitivales en moyen français, Martineau (1990: 70 sqq), qui adopte la représentation de Pollock (c.à.d. (la)), tout en conservant la terminologie Infl au lieu de T de celui-ci (pour la clarté nous utiliserons $\mathrm{T}$ ), suggère que le passage de l'ordre ne $V$ pas à ne pas $V$ reflète un changement paramétrique dans les traits de Agr, changement correspondant au passage de la flexion verbale riche de l'ancien et du moyen français à une flexion d'une moindre richesse. Plus précisément, Agr serait passé de la spécification [+pronominal], qui encodait au départ l'idée traditionnelle que le sujet nul était légitimé par une flexion riche à valeur pronominale, à la spécification [-pronominal]. Tout comme le propose Rochette (1988), Martineau défend l'idée que Agr riche avait la capacité :

1. de légitimer des sujets nuls (p. 70-71),

2. de légitimer des infinitifs substantivés (p. 71-73);

3. de L-marquer le VP infinitif, avec pour effet d'enlever à VP son statut de barrière inhérente (p. 70,74 sqq).

La troisième propriété est tenue pour responsable de deux phénomènes au moins : d'une part, elle intervient dans la possibilité de la montée longue du clitique objet hors de la proposition infinitivale, comme le propose Kayne (1989) pour l'italien; d'autre part, c'est cette capacité de L-marquer le VP qui permettrait, à l'instar de ce que suggérait Pollock 
(1989: 412) pour l'italien, l'extraction du verbe hors de VP, son déplacement dans Agr puis dans T, AgrP n'héritant pas du statut de barrière d'un VP L-marqué (Martineau 1990 : 76, 201 sv.). La perte du trait [+pronominal], située par Martineau (1990: 130 et 207) à l'époque du français classique, sans empêcher la sortie de V hors du VP jusqu'à Agr, aurait rendu impossible la montée du verbe au-delà de AgrP, cette catégorie acquérant désormais pour elle le statut de barrière par héritage de VP. L'effet attendu est dès lors que les particules pas et point précèdent toujours le verbe à l'infinitif, ces éléments se situant à gauche de Agr dans la représentation (la) adoptée par Martineau.

En référence à la possibilité pour les modaux et les auxiliaires d'apparaître devant le forclusif en français moderne, Martineau adopte la suggestion de Pollock concernant l'opacité de T[-fini] à la transmission des rôles thêta: le mouvement des auxiliaires jusqu'à $\mathrm{T}$ (contrairement à celui des verbes pleins) serait permis parce que ces verbes n'assignent pas de rôles thêta.

Bien que cette analyse couvre à beaucoup d'égard les faits tels qu'ils sont décrits, un certain nombre de questions nous font penser que divers aspects de celle-ci devraient être revus. D'une part, plusieurs arguments de nature théorique invoqués pour expliquer le caractère légitime ou illégitime de certaines structures nous semblent sujets à controverse. D'autre part, quelques corrections nous semblent devoir être apportées à la datation de certains phénomènes, corrections qui peuvent amener à mettre en doute l'existence d'une corrélation entre certains des changements notés. Malgré tout, il ne faut pas perdre de vue que même si les arguments théoriques donnés à l'appui de certaines représentations peuvent être contestés, il est possible que les représentations ellesmêmes soient effectivement correctes.

\subsection{Arguments théoriques}

Un premier point qui ressort de l'analyse proposée est qu'en fait, elle fait appel à l'effet conjoint de deux facteurs pour rendre compte de la disparition progressive de la montée $\mathrm{du}$ verbe plein dans T[-fini] (situé pour Martineau, rappelons-le, plus haut que Agr et Neg), l'un explicite, mettant enjeu la théorie des barrières, comme nous l'avons vu, l'autre plus implicite, mettant en jeu la théorie des rôles thêta. Etant donné le recours à la théorie des rôles thêta pour rendre compte des différences de distribution des verbes pleins d'une part et des auxiliaires et des modaux de l'autre par rapport à pas en français contemporain, on semble être amené à conclure qu'en moyen français, T n'aurait pas été opaque, puisque les verbes pleins pouvaient apparemment occuper la position T. Il faut donc que $\mathrm{T}$ soit devenu opaque à la transmission des rôles thêta, et en vertu de cela, un domicile illégitime pour les verbes pleins, indépendamment du statut de barrière que AgrP aurait pu acquérir. En fait, malgré le statut présumé de barrière par héritage acquis par AgrP à partir du moment où Agr serait devenu [-pronominal], ce statut est mis en doute par le fait que le changement invoqué n'a pas empêché les auxiliaires et les modaux de continuer à apparaître à gauche de pas, c.à.d. de monter à $\mathrm{T}$ dans cette analyse. En bref, l'hypothèse selon laquelle AgrP serait devenu une barrière ne parait pas correcte, et elle ne parait jouer aucun rôle à partir du moment où l'approche de Pollock en termes de rôles thêta est invoquée. ${ }^{9}$

30 Depuis les suggestions de Belletti, il y a consensus, du moins pour ceux qui tiennent Agr associé au sujet pour une tête syntaxique, que Agr commande T. Dans cette perspective, on pourrait conserver l'esprit de l'analyse de Pollock et supposer que la catégorie 
fonctionnelle devenue opaque à la transmission des rôles thêta dans les infinitivales serait Agr et non T. Une approche reposant sur la théorie des rôles thêta, en liaison avec un changement dans le statut de Agr, nous semble toutefois problématique à divers égards.

\subsection{Absence de corrélation entre les divers phénomènes}

D'une manière générale, si on considère la figure 1 et les faits décrits ci-dessous, il nous paraît difficile d'envisager que l'évolution observée soit due simplement à un seul changement paramétrique dont la conséquence nécessaire aurait été l'émergence, à une même époque, d'une constellation de nouvelles caractéristiques dans la langue, notamment l'impossibilité de sujets nuls, l'interdiction de la montée longue du clitique objet, la disparition des infinitifs substantivés, et la perte pour le verbe plein, mais pas pour les auxiliaires ou les modaux, de la possibilité d'apparaitre à la gauche de pas.

Considérons la chronologie. L'évolution du français vers une langue qui n'admet plus les sujets nuls est complétée vers la fin du XVIe siècle (cf. Depuis, Lemieux, et Gosselin 1992), alors que la réalisation du verbe plein à l'infinitif à la gauche de pas est encore attestée avec une certaine vigueur durant la première moitié du XVIIe siècle et ensuite dans une mesure progressivement moindre, jusque vers la fin de la première moitié du XVIIIe siècle, les deux ordres se retrouvant d'ailleurs chez les mêmes auteurs, comme le montre le contraste entre les exemples en (a) et (b) ci-dessous.

\begin{tabular}{|l|l|}
\hline (13) & a ...n'êtes-vous pas malheureux de n'avertir pas votre maitre, qui \\
\hline & $\begin{array}{l}\text { peut-être ne s'y connaît pas, qu'il se fait tort de n'avoir pas de bon vin à donner à ses } \\
\text { amis ? (Tallemant des Réaux [1657-1669] CDR, p. } 89 \text { ). }\end{array}$ \\
\hline & $\begin{array}{l}\text { a' Le cardinal pourtant n'était guère bien informé des choses, de ne savoir pas ce qu'on } \\
\text { faisait de l'argent, (Tallemant des Réaux CDR, p. 62) }\end{array}$ \\
\hline $\begin{array}{l}\text { b Le cardinal, [...], porta ce respect au roi que de ne pas défaire ce qu'il avait fait. } \\
\text { (Tallemant des Réaux CDR, p. 71) }\end{array}$ \\
\hline $\begin{array}{l}(14) \\
\text { a ...afin que vous appreniez à ne présumer pas de vous-mêmes. (Bossuet, Discours, 1682, } \\
\text { p. 252) }\end{array}$ \\
\hline $\begin{array}{l}\text { b ...ils aimaient mieux endurer toutes sortes d'extrémités, que de ne pas honorer Jésus- } \\
\text { Christ (Bossuet, Discours, 1682, p. 138) }\end{array}$ \\
\hline $\begin{array}{l}\text { (15) } \\
\text { a J'avais trop envie d'être illustre, pour n'écouter pas ce conseil... (Lesage [1668-174 ?], } \\
\text { Gil Blas, 1715-1735, p. 148) }\end{array}$ \\
\hline $\begin{array}{l}\text { b ...je sais trop bon gré à mon étoile [...] pour ne pas jouir de ma bonne fortune... (Lesage, } \\
\text { Gil Blas, 1715-1735, p. 28) }\end{array}$ \\
\hline
\end{tabular}

On trouve cette construction jusqu'à nos jours chez quelques rares auteurs chez qui il doit s'agir d'un vestige "élégant " (le plus récemment, à notre connaissance, chez Maurice Rat, (ex. 22c)). Nous reviendrons sur ce fait plus loin. 
Si la disparition des sujets nuls semble bien liée à l'appauvrissement de Agr, il ne semble pas que cet appauvrissement ait eu de conséquence radicale et immédiate sur la position du verbe plein. En particulier, il ne semble pas qu'il ait entraîné de restriction sur le déplacement du verbe plein, si les exemples de type $n e V_{\text {infn }}$ pas doivent effectivement s'expliquer, pour ces verbes, par un déplacement de $\mathrm{V}$ dans Agr. De même, l'appauvrissement de la flexion ne semble pas avoir eu d'effet radical sur la montée longue du clitique objet : cette construction est en régression constante depuis le début du moyen français, les classes de verbes qui l'acceptent allant en diminuant, mais on la retrouve encore jusqu'à la fin du XVIII siècle avec les modaux, après la disparition de la construction du verbe plein infinitif à gauche de pas et longtemps après la disparition des sujets nuls (et par la suite encore, mais de manière relativement rare, cfr. Galet 1971, Martineau 1990). En l'absence d'étude détaillée sur l'histoire des infinitifs nominalisés du français, il est difficile d'en dire quelque chose avec confiance, mais nos dépouillements nous laissent croire qu'il n'est pas impossible que cet emploi disparaisse de manière productive en même temps que la possibilité de sujets nuls. D'une manière plus générale, la chronologie des changements relatifs aux sujets nul, à la montée longue du clitique, et à la présence du verbe plein à gauche de pas suggère que tous ces changements ne sont pas étroitement liés. Nous verrons plus loin qu'il n'est cependant pas impossible d'imaginer un scénario permettant de lier la disparition des sujets nuls et la disparition postérieure de la construction ne $V_{\text {infn }}$ pas pour les verbes pleins, après une période où la construction ancienne et la construction qui devait finir par l'emporter sont en variation.

Par ailleurs, au sujet du lien éventuel entre l'appauvrissement de la richesse de la flexion et la disparition de la construction $n e V_{\text {infn }}$ pas pour les verbes plein, il faut noter que ce n'est pas le cas que tous les auteurs qui permettent les sujets nuls fassent un usage régulier de la construction $n e V_{\text {infn }}$ pas dans le cas des verbes pleins, bien que cette corrélation soit presque toujours présente. Ainsi, Vigneulles (1471-1528) a une grammaire avec sujets nuls, montée longue du clitique, et infinitifs nominaux, mais dans les deux longs textes de lui que nous avons étudiés, les Cent Nouvelles Nouvelles (CNNV) et son Journal, on ne note que deux cas de verbe pleins à gauche de pas ou point, ${ }^{10}$ contre cinq avec le verbe à droite (six si l'on compte l'exemple (17f) où ne n'est pas dans la même proposition que point) : ${ }^{11}$

\begin{tabular}{|c|c|}
\hline$(16)$ & $\begin{array}{l}\text { a ...afin de n'aler point avec les aultres audit royaume de Naples (Vigneulles, CNNV } \\
99 / 61 \text { ) }\end{array}$ \\
\hline & $\begin{array}{l}\text { b jaisoit ce que le roy des romains feignoit de ne s'en meller point (Vigneulles, Journal, } \\
\text { p. 147) }\end{array}$ \\
\hline \multirow[t]{3}{*}{$(17)$} & a ...et ainsi aurez excuse de n'en point donner. (Vigneulles, CNNV, 52/95) \\
\hline & b ...car par ainsi pourrés estre quicte de n'en point donner. (Vigneulles CNNV, 52/115) \\
\hline & $\begin{array}{l}\text { c ... mais il faindoit de n'en point savoir et aussi la marchande n'en sçavoit rien; } \\
\text { (Vigneulles, CNNV, 38/16) }\end{array}$ \\
\hline & d ... ils étaient délibérés de ne point rendre le prisonnier... (Vigneulles, Journal, p. 92) \\
\hline
\end{tabular}


e toutes ces choses faixoient par malvitiés faindant de ne point congnoistre la plaice. (Vigneulles, Journal, p. 68)

f je luy rescrips que mon intencion n'estoit de encor point retourner. (Vigneulles, Journal, p. 18)

D'autre part, alors que le verbe plein cesse d'apparaître de manière fréquente à la gauche de pas au cours de la deuxième moitié du XVIIe siècle, le clitique seul devant le forclusif est attesté avec vigueur tout au long du XVIII ${ }^{e}$ siècle pour l'ensemble des clitiques et ce phénomène se poursuit encore durant le $\mathrm{XIX}^{\mathrm{e}}$ siècle pour en et pour $y$. De même, la montée du clitique devant le verbe modal dans les constructions à restructuration, courante au XVIIe siècle, se poursuit dans une certaine mesure pendant tout le XIX ${ }^{\mathrm{e}}$ siècle. Le changement d'un seul paramètre, la perte du statut pronominal de Agr, n'a pu faire de AgrP, dans une description du type de (1a), une barrière au mouvement du verbe et des clitiques.

37 L'approche en termes de barrières apparaît donc problématique en ce qui concerne l'évolution de la position relative du verbe plein et des clitiques par rapport à pas et point. Notons d'ailleurs, de manière anecdotique du fait de la rareté du phénomène dans notre corpus, deux exemples avec un clitique de part et d'autre du forclusif (18a-b).

\begin{tabular}{|l|l|}
\hline (18) & a Et pourquoi ne me pas l'avoir dit ? (Diderot, Jacques, 1784, p. 157) \\
\hline & $\begin{array}{l}\text { b -je vous prie tous deux de ne vous point en aller qu'on ne m'ait apporté mon habit, ... } \\
\text { (Molière, Bourgeois G., 1671, p. 9) }\end{array}$ \\
\hline
\end{tabular}

Clairement, si la présence du clitique à droite du forclusif était due à une barrière l'empêchant de se déplacer plus haut dans la structure, il n'y aurait pas simultanément un clitique à gauche du même forclusif. Ces exemples pourraient s'analyser par le déplacement d'un seul clitique par-dessus la négation, ce qui n'est pas sans rappeler les cas de divorcedesclitiques(cliticsplitting),qu'on trouve notamment en français au XVIIe siècle (Galet 1971) ainsi qu'en vaudois (Laenzlinger 1990, où un sous-ensemble des clitiques associés au verbe subordonné se trouve devant le verbe principal (19)).

\begin{tabular}{|l|l|}
\hline$(19)$ & a Je me veux toutefois en venger par malice (Corneille, Mélite, 1629, v. 1015) \\
\hline & b Je le veux vous montrer (Laenzlinger, 1990, p. 12, parallèle à Kayne 1989, p. 248) \\
\hline
\end{tabular}

La corrélation entre les divers phénomènes ne paraissant donc pas aussi étroite qu'il a pu le sembler, nous allons explorer une autre approche. 


\section{Hypothèses alternatives}

\subsection{La proposition infinitive négative}

Nous adoptons une représentation du type de (20) comme hypothèse de travail sur la structure des propositions infinitives négatives.

(20)

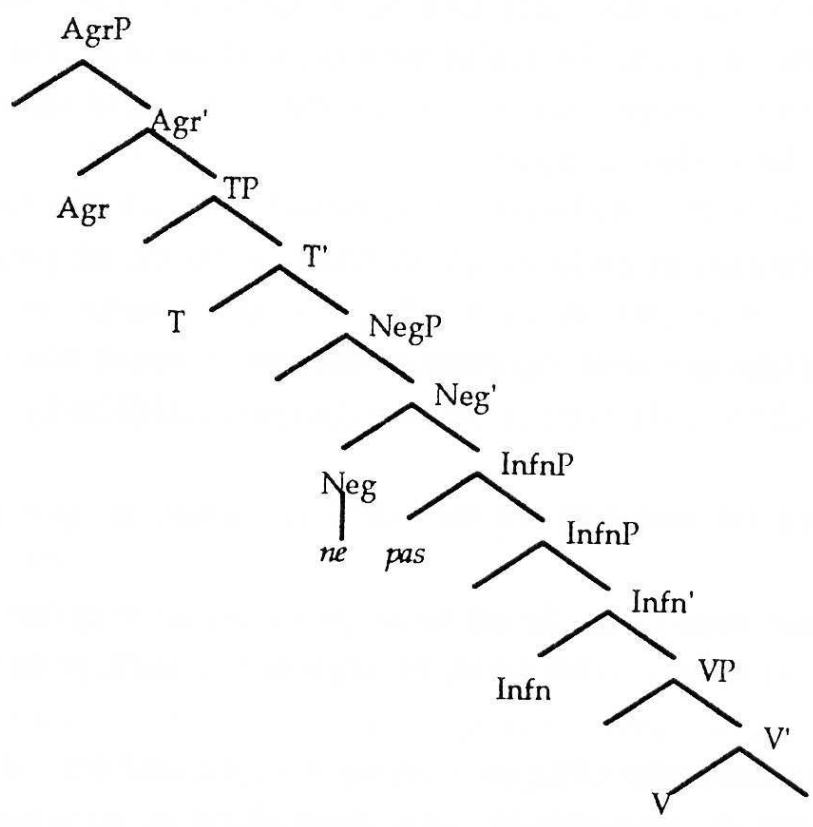

Comme Pollock, nous supposons que la structure des infinitivales est minimalement distincte de celle des phrases à temps conjugué, mais contrairement à lui et à Belletti, qui conçoivent l'opposition temps conjugué vs infinitif en termes des traits [+fini] vs [-fini] associés au nœud T, nous adoptons la suggestion de Kayne (1990) selon laquelle la morphologie infinitivale correspond à une tête morphosyntaxique Infn distincte de T. Nous conservons toutefois l'idée de la présence d'un nœud T. L'existence éventuelle d'un nœud Agr dans les infinitives est immatérielle pour les faits qui nous intéressent; dans la mesure où Agr est présent, il devrait c-commander T, comme l'a proposé Belletti (1990). Enfin, nous retenons l'hypothèse de l'existence d'une catégorie NegP dont la tête est ne, mais nous la situons plus bas que TP, contrairement à Belletti. Par ailleurs, pour le français contemporain, nous considérons que pas n'est pas localisé dans SpecNegP, mais qu'il est adjoint au VP maximal gouverné par ne en D-structure (pour des arguments dans ce sens, voir Hirschbühler et Labelle 1993a).

Cette structure nous servira de point de référence pour les faits d'évolution, modulo les ajustements éventuels requis par la syntaxe propre des époques concernées.

Etant donné les observations que la figure 1 a permis de faire, trois scénarios au moins sont imaginables pour rendre compte des changements observés.

1. Les changements observés résultent de la disparition progressive de la montée de $\mathrm{V}$ à $\mathrm{T}$. Il faut apparemment postuler ici trois changements grammaticaux indépendants, dont la nature est à déterminer. Un premier changement aurait favorisé la disparition progressive de montée de $\mathrm{V}$ à $\mathrm{T}$ pour les verbes pleins; plus tard, un autre changement aurait conduit à la même chose pour les modaux; et le dernier changement serait reflété 
par une diminution marquée de la montée de être et avoir, diminution qui aboutira peutêtre à la disparition de toute construction avec le verbe à l'infinitif à gauche de pas.

2. Les changements observés ne sont pas l'effet de changements dans la position du verbe, mais de changements dans la position de pas.

3. Certains changements sont à imputer à la première hypothèse, alors que d'autres le sont à la seconde.

Dans une série d'exposés, nous avons initialement exploré la première hypothèse et suggéré que les changements d'ordre de mots notés successivement pour les verbes pleins, les modaux et les auxiliaires reflétaient le même type de phénomène: la disparition progressive de la montée de verbe, qui aurait affecté les trois classes de verbes à des moments différents. Pour chaque classe de verbe, le déplacement de V à Agr (pardessus pas), d'obligatoire qu'il était, serait devenu facultatif. Un principe d'économie dérivationnelle n'aurait plus laissé subsister, avec le temps, que la variante sans montée de verbe.

Si nous continuons de penser que le changement noté dans les exemples avec auxiliaires ou modaux est bien un changement dans l'étendue du déplacement du verbe, nous pensons que dans le cas des verbes pleins, une analyse alternative reposant sur un changement dans la position de pas et point n'est pas dépourvue d'attraits. Dans ce qui suit, nous présentons et discutons les deux façons de voir le changement d'ordre mettant en jeu les verbes pleins.

\subsection{Changement dans le mouvement de $\mathrm{V}$ plein}

\subsubsection{Appauvrissement de Agr}

Il est naturel d'envisager l'hypothèse, en l'absence évidemment de faits qui suggèrent le contraire, que la représentation hiérarchique de la D-structure d'une infinitivale est constante à travers l'histoire, et que l'ordre ne $V$ pas caractéristique des données les plus anciennes résulte du fait que le verbe, ayant acquis sa morphologie infinitivales dans Infn, s'est déplacé plus haut que NegP, c'est-à-dire minimalement à $T$. En d'autres mots, dans la période en question, le verbe infinitif et le verbe à temps conjugué se déplacent plus haut que NegP. A cet égard, le moyen français se comporterait en grande partie comme l'italien contemporain, si l'on s'en rapporte à l'analyse suggérée par Belletti (1990, p. 73 et 70) pour rendre compte des exemples du type de (21). ${ }^{12}$

\begin{tabular}{|l|l|}
\hline$(21)$ & $\mathrm{a}$ *Gianni ha deciso di non più tornare \\
\hline & b Gianni ha deciso di non tornare più \\
\hline & G. a décidé de ne plus revenir \\
\hline
\end{tabular}

Partant de l'hypothèse de travail qu'un appauvrissement de Agr joue un rôle dans le passage de l'ordre ne $V$ pas à ne pas $V$, les remarques de la section 4.3. amènent à dire que ce changement n'a pas créé d'interdit pour l'ordre ne $V$ pas, mais qu'il a favorisé l'ordre alternatif ne pas $V$, et cela pour les verbes pleins uniquement. Etant donné le caractère véritablement exceptionnel de l'ordre ne pas $V$ durant les XVe et XVIe siècles, nous pouvons supposer (en idéalisant peut-être) que l'ordre ne $V$ pas était initialement 
obligatoire. Admettons que cet ordre reflète le déplacement de V à Agr, Agr riche rendant obligatoire le mouvement du verbe. ${ }^{13} \mathrm{Il}$ serait alors possible que l'affaiblissement de Agr ait fait disparaître le caractère obligatoire de la montée du verbe infinitif à cette position.

Supposons qu'il en est ainsi. A quoi correspondrait exactement l'affaiblissement de Agr, responsable du caractère pro-drop de la langue? Selon Dupuis, Lemieux et Gosselin (1922), Agr aurait eu, en ancien français, la spécification [a personne, b pluriel]; ces auteurs attribuent l'affaiblissement de Agr à la disparition du trait [b pluriel] de son système de spécification. Faisons l'hypothèse supplémentaire que le trait [a personne] n'est présent que dans le Agr des phrases à temps conjugué. En conséquence, l'Agr des propositions à l'infinitif serait passé de la spécification [b pluriel] à l'absence de toute spécification pour la personne et pour le nombre. Le développement dans les infinitivales d'un Agr dépourvu de toute spécification de nature morphologique a pu constituer le changement grammatical initial à partir duquel la montée du verbe à Agr n'est plus obligatoire. Cette hypothèse rejoint celle de Lois (1989: 55ss) pour les langues romanes et celle de Vikner $(1991$ : 138) pour les langues germaniques qui ont connu ce changement. Etant donné que le $\mathrm{T}$ des infinitivales est lui aussi morphologiquement nul, nous nous retrouvons dans une situation ou, en principe, le verbe peut rester dans Infn et sous la négation, ou monter par-dessus la négation jusqu'à $\mathrm{T}$ (et éventuellement jusqu'à Agr). Comme l'on note que, initialement, seuls les verbes pleins semblent entrer dans la nouvelle construction, on supposera que quelque chose de particulier se passe dans le cas des auxiliaires et des modaux, qui fait qu'ils continuent à monter obligatoirement plus haut que Infn; nous reviendrons sur ce point.

\subsubsection{Economie}

49 Nous limitant dans un premier temps aux verbes pleins, il s'agit d'expliquer la diminution progressive de la montée de ce type de verbe, étant supposé que la montée était devenue facultative. Une idée traditionnelle qui vient à l'esprit est celle d'économie, exploitée récemment par Chomsky (1991). Nous dirons que si deux dérivations syntaxiques sont permises, toutes choses étant égales par ailleurs, la dérivation syntaxique la plus courte est favorisée. Un point important de cette proposition très générale est évidemment la condition toutes choses étant égales par ailleurs.

Cette condition jouera un rôle important dans l'explication du comportement des auxiliaires et des modaux. Nous verrons d'abord comment elle peut être exploitée pour rendre compte du caractère progressif de la disparition de la montée du verbe plein. En effet, les données primaires accessibles à l'apprenant représentent un facteur qui fait que toutes choses ne sont pas égales par ailleurs. Au début de la période où le caractère obligatoire de la montée de $\mathrm{V}$ infinitif vient de disparaître, l'enfant qui acquiert sa langue est confronté à une situation où le verbe plein apparait pratiquement toujours à gauche de la négation. Il n'y a pas de raison de penser qu'il s'agit là d'une donnée positive qui puisse être ignorée et l'apprenant développera donc une grammaire permettant la montée du verbe au-delà de Neg jusqu'à $T$ et éventuellement Agr. Mais cette montée ne serait plus forcée par le besoin de satisfaire à un impératif grammatical qui serait fonction de la nature de Agr. Cette situation permettrait au principe d'économie dérivationnelle de se manifester, éventuellement en conjonction avec d'autres facteurs, avec pour effet une diminution progressive de la proportion de verbes pleins à gauche du forclusif, le choix entre montée et absence de montée pouvant faire l'objet d'une 
exploitation stylistique. ${ }^{14}$ Avec le temps, on en arriverait à une proposition suffisamment faible de verbes montés au-dessus de la négation pour que l'enfant ne considère pas qu'il est nécessaire de les intégrer à sa grammaire.

51 En résumé, la montée du verbe plein serait finalement exclue sur la base de l'extrême rareté des données positives accessibles à l'enfant, en conjonction avec le principe d'économie : lorsque deux dérivations sont permises, si l'une est bien représentée dans les données primaires et résulte de la dérivation la plus courte en syntaxe, alors que l'autre ne fait pas partie des données et représente la dérivation la plus longue, cette dernière dérivation est marquée comme exclue. Reste alors à rendre compte des survivances, chez des auteurs comme Gide, Montherlant, et Maurice Rat.

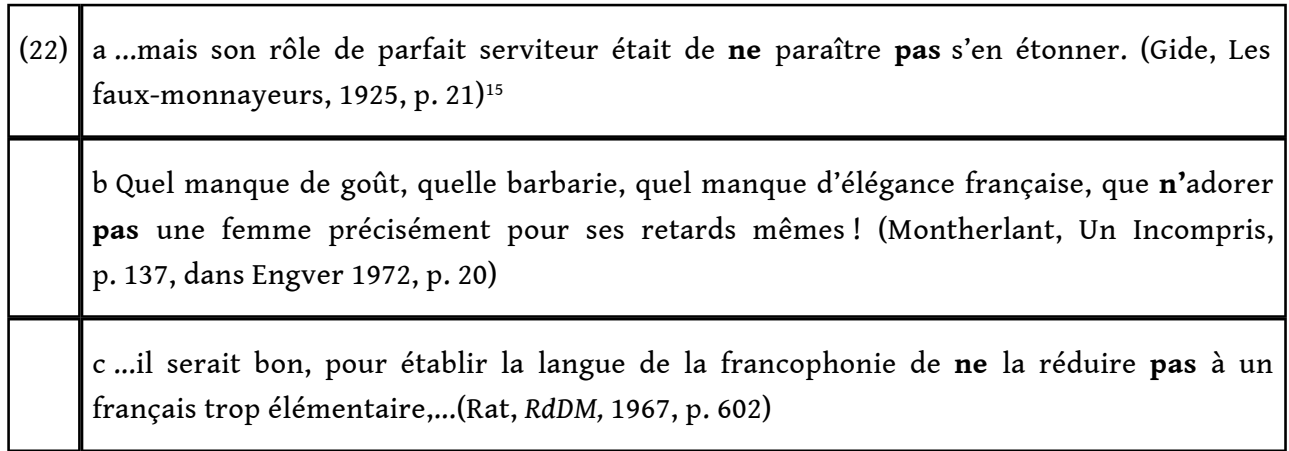

52 Etant donné le caractère exceptionnel de ces données, et ce qu'on sait des auteurs chez qui on les note, il n'est pas impossible qu'il s'agisse là de calques basés sur leur familiarité avec la tradition littéraire. La question qui se pose évidemment concerne la manière dont ces formes sont analysées dans la langue de ces auteurs archaïsants.

\subsection{Changement dans la position du forclusif}

53 Une hypothèse alternative à celle qui vient d'être décrite est que le passage de $n e V$ pas à ne pas $V$ pour les verbes pleins reflète simplement un changement dans la position d'attachement des forclusifs comme pas, point, mie. Dans cet esprit, la structure d'une infinitivale négative aux XVe-XVIe siècles n'aurait pas été du type de (20), mais plutôt du type de (23a), avec les éléments comme pas adjoints à VP plutôt qu'à InfnP ; cette structure aurait été concurrencée puis supplantée par (20), reproduit ici en (23b). 
(23) a

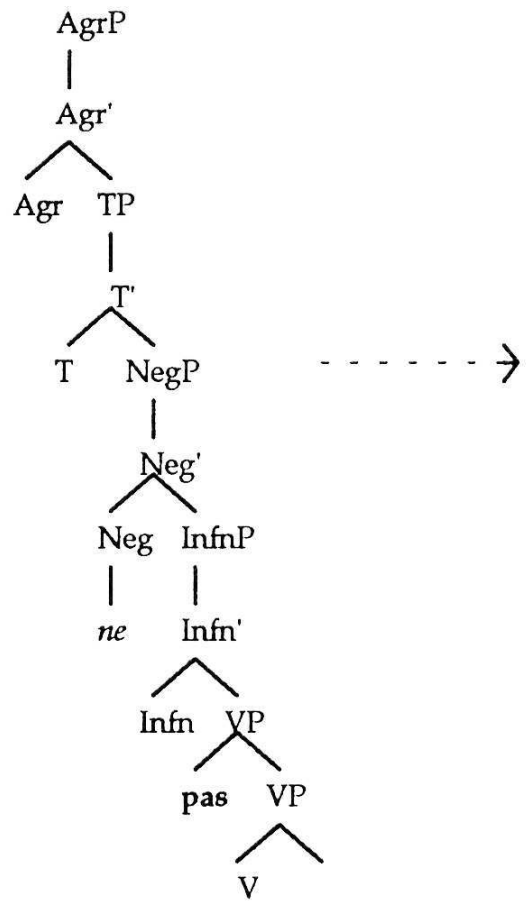

(23) $b$

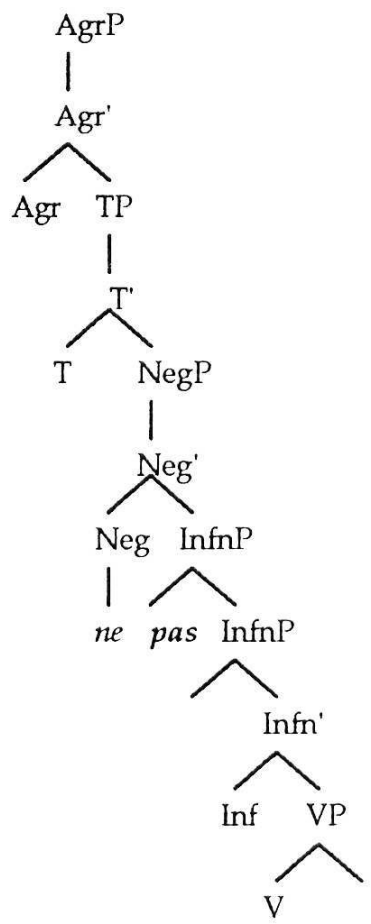

En fait, Pollock (1988 : 412-414 et note 10 p. 373) lui-même, sur la base des jugements en (24), analyse (24b) comme un cas où point, plus, rien seraient en position de spécifieur d'un NegP adverbial engendré en position initiale de VP (et dont ne serait toujours la tête).

Selon les jugements dont Pollock fait état, point,pluset rienpourraient non seulement précéder le verbe plein à l'infinitif, tout comme pas,mais également le suivre, contrairement à pas. La plupart des locuteurs considéreraient les exemples de (24b) comme parfaits, avec, dans le pire des cas, une certaine résonance littéraire, signalée par le point d'interrogation facultatif. Notre analyse (23a) se rapproche de la sienne en ce qu'elle considère que certains forclusifs ont pu être engendrés comme adjoints à VP. Toutefois les jugements donnés en (24) ne correspondent ni à notre expérience ni à l'image que l'on peut se faire du français contemporain littéraire sur la base des textes.

\section{Changement dans la position de $V$ ou de pas?}

Peut-on choisir entre l'analyse en termes de changement dans l'étendue du mouvement de verbe décrite à la section 5.2 et l'analyse en terme de repositionnement de pas illustrée par les arbres (23a-23b) ? Notons que dans le cadre de l'analyse qui vient d'être suggérée, on ne s'attend pas à ce qu'il y ait un lien quelconque entre le changement dans l'ordre relatif du verbe infinitif et du forclusif d'une part et le changement dans la richesse de la flexion postulé pour rendre compte de la disparition des sujets nuls. De même, cette 
analyse ne suggère pas de lien avec la disparition de la montée du clitique. Ceci n'est pas une mauvaise chose puisque nous avons vu que ces divers changement se produisent à des moments différents.

L'analyse suggérée ici serait appuyée par l'existence, à l'époque du changement ne $V$ pas ${ }^{\circledR}$ ne pas $V$, d'autres changements dans la grammaire de la négation et des forclusifs. Il nous semble qu'il en existe.

58 Avant le changement dans les positions respectives du verbe plein à l'infinitif et du forclusif, et encore dans une certaine mesure après, ne pouvait à lui seul exprimer la négation. A l'origine, il semble que pas et point fonctionnaient comme des quantifieurs exprimant une quantité minime (Muller, 1991, Chap. V.II et Englebert 1982'). Selon Muller, ces termes pouvaient fonctionner comme des quantifieurs portant sur le verbe, la négation de ceux-ci permettait d'exprimer la quantité nulle. Dans cette perspective, il paraît naturel que, comme quantifieurs associés au verbe, ces éléments soient engendrés comme adjoints à VP. De plus, pas et point pouvaient être utilisés comme termes de polarité négative, emploi qui disparait apparemment au XVIIe siècle (Muller, 1991 : 226). Par exemple, on trouve pas et point sans ne dans une question « orientée vers une réponse négative" (Muller, 1991: 25), alors qu'une question négative est orientée vers une réponse positive. Ainsi, Muller glose l'exemple (25) par « Suis je si peu que ce soit plus à mon aise que toy?», le contexte indiquant que la réponse attendue est non, le locuteur ayant été soumis à la torture :

(25) Et moy, suis-je dans un bain? suis-je pas plus à mon aise que toy ? (Montaigne, Essais, III, 6)

A côté de cet emploi de pas/pointcomme termes de polarité négative, il y a évidemment des emplois indéniablement purement négatifs puisque c'est au XVIe siècle que l'on note les premiers emplois de pas/pointutilisés sans nepour exprimer la négation de phrase dans les assertions (Muller, $1991: 224) .{ }^{17}$ Il ne serait donc pas surprenant que la réanalyse de paset pointcomme mots purement négatifs ait entraîné à terme un changement dans leur position qui les aurait rapprochés de ne.

60 Finalement, d'autres facteurs ont pu favoriser, de manière additionnelle peut-être, le changement de position de pas et point. Ainsi, un examen préliminaire de la distribution de jamais et rien montre que, chez certains auteurs au moins, ces termes se retrouvent parfois à la gauche du verbe infinitif alors que pas et point ne s'y retrouvent jamais. Il ne serait pas impossible que les locuteurs aient étendu à pas et point une des possibilité de placement de jamais et rien.

\section{Les modaux et les auxiliaires}

61 L'analyse que nous venons de proposer pose le problème des auxiliaires et des verbes modaux. Si pas a commencé à se placer à la droite immédiate de NegP dans le cas des verbes infinitifs, il n'y a pas de raison de supposer qu'il en soit autrement dans le cas des modaux et des auxiliaires. Puisque ces derniers apparaissent néanmoins à gauche de pas après le changement observé dans la position relative de celui-ci et des verbes pleins, ce doit être parce qu'ils se trouvent plus haut que Neg, c'est-à-dire au minimum dans T, 
alors que ce n'est pas le cas pour les verbes pleins. Comment peut-on expliquer ce fait, ainsi que les changements ultérieurs?

Nous avons accepté jusqu'ici sans discussion la présence d'un nœud $\mathrm{T}$ dans les infinitivales. Comme nous allons en faire un usage crucial pour expliquer certains faits, il peut être utile de justifier l'existence de $\mathrm{T}$ dans ce type de proposition, d'autant plus que certaines approches la mettent en doute (nous restons agnostiques quant à la présence de Agr dans les infinitivales).

Bouchard (1992) présente T non comme l'expression du temps, mais comme le point d'ancrage de Temps au moment d'énonciation (« the anchor of Tense on to the Moment of Speech») et considère que $\mathrm{T}$ n'est pas présent dans les infinitivales. En fait, la caractérisation de $\mathrm{T}$ doit être plus générale, pour permettre l'ancrage du temps dans les subordonnées tensées, où parler en termes de moment d'énonciation n'est pas approprié (Enç 1987). Si T est conceptuellement requis dans les subordonnées tensées pour rendre compte de l'interprétation, notamment en liaison avec les phénomènes de concordance de temps, il nous semble que les mécanismes d'interprétation temporelle justifient également la présence de $\mathrm{T}$ dans les infinitivales. ${ }^{18}$ De plus, la présence, à certaines époques, de clitiques et d'un auxiliaire ou d'un modal entre ne et le forclusif indique qu'il existe une position fonctionnelle d'attachement pour ces éléments dans les infinitivales, position que nous identifions à $\mathrm{T}$ (cf. Laenzlinger (1990) pour une justification intéressante de $\mathrm{T}$ comme lieu d'attachement privilégié des clitiques verbaux).

Admettant la présence de $\mathrm{T}$ dans les infinitivales, envisageons le cas des auxiliaires. Puisque ces éléments peuvent entrer en jeu dans l'expression du temps (après avoir mangé..., je regrette d'être arrivé à cinq heures), il est tentant de spéculer que la montée de l'auxiliaire infinitif jusqu'à $\mathrm{T}$ est cautionnée par son rôle dans l'interprétation temporelle. Les emplois non temporels de être et avoir auraient le même comportement parce que, malgré les différences d'emploi, il s'agit toujours des mêmes verbes ${ }^{19}$. Des effets d'une analogie purement superficielle ne sont pas à écarter non plus.

Qu'en est-il des modaux ? Ce que nous avons à dire ici est beaucoup plus programmatique. Il a parfois été proposé que le complément infinitival d'un verbe modal est un VP. L'existence des faits suivants suggère que le complément infinitif des verbes modaux projette au contraire au moins jusqu'à $\mathrm{T}$ :

\begin{tabular}{|l|l|}
\hline$(26)$ & a puis que pour vouloir n'avoir pas présenté ... (Bayle CPPJC, 1686) \\
\hline \hline & $\begin{array}{l}\text { b je la supliois de vouloir bien ne me pas engager (Chasles, Les illustres françoises, 1713, } \\
\text { p. 298) }\end{array}$ \\
\hline & $\begin{array}{l}\text { c je veux, enfin, pouvoir ne me pas moins tromper Crébillon fils, Lettres athéniennes, } \\
1771, \text { p. 183) }\end{array}$ \\
\hline & \begin{tabular}{l} 
d je crus devoir n'en plus parler (Mme Riccoboni, Jenny, 1764,p. 419). \\
\hline
\end{tabular}
\end{tabular}

Nous admettrons aussi, à la suite de beaucoup d'autres, que le cadre temporel des compléments infinitifs des verbes modaux est référentiellement dépendant de celui du verbe modal : l'infinitif place le moment d'évaluation de la proposition dans laquelle il se trouve à un moment qui se trouve dans un futur possible par rapport au temps de la 
principale. (Stowell 1982). Certaines analyses expriment cette dépendance du temps de la subordonnée au temps de la principale au moyen d'un opérateur temporel anaphorique en Comp (Enç 1987, Kempchinsky 1992, Raposo 1987, Stowell 1982, Terzi 1992). Une analyse alternative suppose qu'en présence des verbes modaux une chaîne de dépendance anaphorique est établie entre le $\mathrm{T}$ de la proposition contenant le modal et celui de sa subordonnée infinitivale. Ceci, joint à la coïndexation des sujets des deux propositions, et possiblement à l'absence de complémenteur lexical ou d'autres éléments susceptibles de donner lieu à des violations de la minimalité, rendrait possible la montée longue du clitique (Kayne 1989, Luján 80 Roberts 1992, Terzi 1992). ${ }^{20}$

Dans le cas où le verbe modal est lui-même à l'infinitif, il n'y a pas lieu de supposer que les choses se passent différemment. Il nous faut donc supposer que le $\mathrm{T}$ dominant le verbe modal à l'infinitif est actif au sens où il constitue la tête de la chaîne de temps et où il constitue le point d'ancrage du temps de la proposition subordonnée. Il est très possible qu'étant actif, ce $\mathrm{T}$ ait certaines des propriétés d'un $\mathrm{T}$ [+fini] et attire le verbe modal. ${ }^{21}$

Un facteur supplémentaire qui a pu jouer dans la longévité de la montée de verbe dans le cas des modaux et des auxiliaires est mis en évidence par Santorini (1989: 151-152). Il s'agit d'un facteur prosodique discuté par Wackernagel (1892). Dans plusieurs langues indo-européennes, le verbe fléchi semble agir comme un clitique phrastique qui tend à graviter autour de la seconde position de la phrase. Ceci suggère à Santorini pour l'évolution du Yiddish que dans les subordonnées tensées les verbes "légers »(les auxiliaires et les modaux) auront plus vite tendance à apparaître en position médiane de phrase que les verbes pleins qui eux tendront à rester en fin de proposition. En effet, dans ses données, les modaux apparaissent en deuxième position proportionnellement plus souvent que les auxiliaires, qui eux-mêmes y apparaissent plus souvent que les verbes pleins. Pour le français du XVIII siècle et, dans une certaine mesure, celui du XIX ${ }^{e}$ siècle, nous remarquons que les clitiques peuvent apparaitre devant le forclusif, ce qui nous donne des raisons de croire que cette position est bien une position « Wackernagel » qui pourrait « attirer » les verbes légers, même non tensés, en particulier dans la mesure où ils servent à définir l'interprétation temporelle. Dans le cas du Yiddish, la deuxième position pour le verbe représente une innovation, et les verbes légers semblent être les moteurs du changement, apparaissant dans cette position plus souvent que les verbes pleins. En français, où la position «Wackernagel » constitue un archaïsme, les verbe légers sont les derniers à l'occuper.

\subsection{Abandon des chaînes T-T dans les infinitivales}

Il reste à caractériser l'évolution ultérieure. L'étape suivante, caractérisée par le fait que les modaux à l'infinitif suivent généralement le forclusif, est celle qui ressemble le plus à la langue décrite par Pollock. Seuls les auxiliaires montent encore de façon assez systématique. Comment expliquer le passage à cette étape ? En fait, cette période, qui suit la révolution française, est caractérisée par trois phénomènes qui ne sont peut-être pas étrangers aux faits que nous étudions.

1) La montée longue du clitique (je le veux faire ${ }^{\circledR}$ je veux le faire) semble devenir une construction marquée dès la fin du XVIIIe siècle ;

2) Les clitiques apparaissent de moins en moins à gauche du forclusif (en et $y$ sont ceux qui y apparaissent le plus longtemps ; la construction moderne devient majoritaire dans la 2e moitié du XVIIIe siècle) ; 
3) Les modaux apparaissent de moins en moins à gauche du forclusif (la construction moderne devient majoritaire dans la première moitié du XIXe siècle).

Prenons d'abord la montée longue du clitique. Alors que certaines analyses font du complément des verbes modaux un VP (Picallo 1990, Rochette 1988) ce qui, dans l'hypothèse où les clitiques s'attachent à la projection fonctionnelle la plus haute (Kayne 1990, 1991), forcerait la montée longue du clitique au T de la principale, nous avons vu qu'il y a des raisons de croire que ce complément est au moins une projection de $\mathrm{T}$. Certains faits notés par Terzi (1992) confirment que ce n'est pas l'absence de temps dans la subordonnée qui rend licite la montée longue du clitique, puisque celle-ci est permise (mais non obligatoire) en Salentino de Brindisi où le complément des verbes modaux est au subjonctif et non à l'infinitif :

\begin{tabular}{|l|l|}
\hline (27) & Lu voggyu kkattu \\
\hline & it-cl I-want-I-buy (Terzi 1992: 159) \\
\hline & Je veux l'acheter \\
\hline
\end{tabular}

71 Si on admet que la montée longue du clitique requiert que le $\mathrm{T}$ de la subordonnée infinitivale soit mis à contribution de manière particulière dans l'établissement d'une chaîne anaphorique avec le $\mathrm{T}$ de la proposition superordonnée - elle n'est permise, par exemple, que si le complément est à l'infinitif ou au subjonctif, mais pas à l'indicatif, et si les deux sujets sont coréférentiels -, la disparition progressive de ce phénomène peut être prise comme un indice que les locuteurs en viennent à minimiser la création de chaînes de $\mathrm{T}$ à $\mathrm{T}$ en syntaxe (en favorisant peut-être le recours au mouvement en LF). La diminution de la montée de clitique jusqu'au verbe principal a peut-être été favorisée par des problèmes d'ambiguité créés par la montée longue du clitique en français, notamment par la confusion entre infinitif et participe passé pour les verbes en -er (je le veux publier) publié) et -ir dont la consonne finale ne se prononçait plus (je le veux fini(r)/fini pour demain) (Galet, 1971). Si la diminution de la montée longue du clitique reflète une tendance à éviter l'établissement de chaînes $\mathrm{T}$ à $\mathrm{T}$ en syntaxe, quelle que soit la raison profonde de ceci, nous aurions là une explication au fait que les modaux infinitifs réduisent massivement leur montée à $\mathrm{T}$ vers la fin du XVIIIe siècle. Ceux-ci étant des verbes assignant des rôles thématiques, ils alignent leur comportement sur celui des verbes pleins. Cette réduction dans le recours à la position structurale définie par T[-fini] a pu amener la diminution progressive à la réalisation du clitique à gauche du forclusif au profit d'un attachement à Infn ou à une de ses projections (Kayne 1990) ou encore à V (Rochette 1988, Martineau 1990).

Des données sur le français du XXe siècle sont disponibles dans l'étude de Engver (1972) qui porte sur la place du verbe dans des textes ayant paru entre 1940 et 1963. Engver ne remarque aucune tendance à faire monter le modal, si l'on exclut un exemple chez Marcel Aymé et quatre chez Montherlant dont la grammaire du placement du verbe infinitif est archaïsante puisqu'il utilise avec abondance la construction ancienne avec le verbe plein : "Quel manque de goût, quelle barbarie, quel manque d'élégance française, que n'adorer pas une femme précisément pour ses retards mêmes!» (Montherlant, Un Incompris, 
p. 137, dans Engver 1972: 20) ${ }^{22}$, construction qui devient caduque, d'après nos données, dans la première moitié du XVIIIe siècle.

Les auxiliaires, quant à eux, continuent de monter de manière très importante pendant tout le XIXe siècle, ce qui indiquerait que le rôle de l'auxiliaire dans l'interprétation temporelle continue pendant un certain temps d'être un facteur favorisant la montée à $T$.

\subsection{Alignement des auxiliaires}

Le français contemporain se caractérise par la diminution graduelle de la montée à $\mathrm{T}$ pour les auxiliaires. Dans l'esprit des hypothèses précédentes, on peut penser que, conformément au principe d'économie que nous avons formulé dans la section 5.2.2., à partir du moment où $\mathrm{T}$ n'est plus associé à un élément lexical pour l'établissement de chaînes temporelles, la tendance à ne plus faire appel à cette position d'une manière générale dans la syntaxe des infinitives s'accentue. Nous supposons que la disparition graduelle de la montée du verbe auxiliaire est liée à un alignement graduel du comportement de ces verbes sur celui des autres types de verbes.

L'étude de Engver (1972, pp. 29 à 34) permet de retrouver pour ces verbes les pourcentages suivants, si l'on exclut des données les exemples trouvés chez Montherlant et Marcel Aymé :

Table 2

$\begin{array}{lrr} & \text { NE PAS AVOIR } & \text { N'AVOIR PAS } \\ \text { +participe } & 34(45 \%) & 41(55 \%) \\ \text {-participe } & 30(71 \%) & 12(29 \%) \\ & & \\ & \text { NE PAS ETRE } & \text { N'ETRE PAS } \\ \text { +participe } & 34(65 \%) & 18(35 \%) \\ \text {-participe } & 55(66 \%) & 28(34 \%) \\ & & \\ \text { Totaux } & 153(61 \%) & 99(39 \%)\end{array}$

On remarque que les verbes avoir et être tendent à suivre le forclusif dans une proportion de 2 pour 1 dans tous les cas, sauf pour le verbe avoir lorsqu'il est employé comme auxiliaire de temps. Dans ce Cas, il apparaît devant le forclusif un peu plus souvent qu'il ne le suit. Cette différence entre avoir auxiliaireet avoir copule est compatible avec notre analyse qui lie la tendance des auxiliaires à monter à $\mathrm{T}$ à leur rôle dans l'établissement de l'interprétation temporelle de la proposition.

Résumons-nous. Une réduction du rôle de $\mathrm{T}$ dans l'établissement de chaines qui favorisaient la montée des modaux a pour conséquence la cessation de la montée des verbes modaux et l'on constate depuis ce temps une diminution progressive de la montée de être et avoir, sans qu'aucun principe véritablement grammatical ne soit en cause, l'économie n'étant à notre sens pas un principe de grammaire. Le comportement de avoir possessif se rapproche plus rapidement de celui des verbes pleins que ne le fait avoir utilisé comme auxiliaire de temps. Si la tendance à la baisse de la montée de l'auxiliaire s'est poursuivie depuis l'étude de Engver et continue son mouvement, on peut s'attendre 
à ce qu'il y ait une étape ultérieure, peut-être déjà réalisée chez certains locuteurs, où aucun verbe ne se placera devant le forclusif.

Notons que, tout comme dans son emploi modal, vouloir dans son emploi non modal monte à $\mathrm{T}$ plus longtemps que les verbes pleins, (...que ne vouloir pas qu'on change? Rousseau $1763 ;$;.. que c'est de ne vouloir pas qu'on démontre! I Diderot, $1784 ;$...accusaient ce parti de ne vouloir point d'assemblée nationale Las Casès 1823), mais dans une mesure qui nous semble moindre qu'avoir ne le fait dans son emploi de copule par opposition à son emploi d'auxiliaire de temps. Nous sommes en train de rassembler l'ensemble des données pertinentes qui nous permettrons de préciser cet aspect dans une étude ultérieure.

\section{Conclusion et remarques sur la motivation du changement}

Nous avons vu qu'il y avait disparition graduelle du caractère obligatoire ou quasi obligatoire de l'ordre ne $V_{\text {Infn }}$ pas, et que celle-ci correspondait à au moins deux types de changements distincts. Dans le cas des verbes pleins, nous avons suggéré qu'ils étaient réalisés dans la position Infn et que la disparition de l'ordre $n e V_{\text {Infn }}$ pas était le résultat d'un changement dans la position de pas. Nous avons attribué le comportement particulier des auxiliaires et des modaux à leur participation à l'interprétation temporelle de leur proposition (les auxiliaires) ou de la subordonnée qu'ils sélectionnent (les modaux). Suite à une désaffection du recours syntaxique à $\mathrm{T}$ dont la cause n'est pas claire, ces verbes s'alignent graduellement sur le comportement des verbes pleins ;

Ceci ne clôt évidemment pas le sujet. De nombreux points pertinents à la discussion n'ont pas été explorés en détail. Ainsi, nous n'avons pas discuté le problème de l'évolution de la place du clitique objet à gauche ou à droite du forclusif. En bref, nous supposons, comme Martineau, que dans le premier cas le clitique est attaché à $\mathrm{T}$, et dans le second à Infn ou directement au verbe. Cette évolution devrait être étudiée dans le cadre d'une étude plus générale de la montée de clitique. De même, nous n'avons pas examiné le comportement des adverbes susceptibles de s'intercaler entre les clitiques et l'infinitif, comme bien, mieux et d'autres ni celui de forclusifs comme plus, jamais et rien, dont l'ordre relatif par rapport aux verbes à l'infinitif n'évolue pas exactement de la même façon que celui des forclusifs discutés ici. Tous ces sujets sont présentement à l'étude.

\section{BIBLIOGRAPHIE}

Belletti, A., 1990, Generalized Verb Movement, Torino, Rosenberg et Sellier.

Bouchad, D., 1992, The Semantics of Grammar. Ms., Montréal, UQAM.

Brunot, F., 1967, Histoire de la langue française des origines à nos jours, T. 2, Paris, Armand Colin. 
Brunot, F., 1966, Histoire de la langue française des origines à nos jours, T. 3, II, Paris, Armand Colin.

Chomsky, N., 1986, Barriers, Cambridge, Massachusetts, MIT Press.

Chomsky, N., 1991, Some Notes on Economy of Derivation and Representation, dans R. Freidin (ed.), Principles and Parameters in Comparative Grammar, Cambridge, Massachusetts, MIT Press, pp. 417-454.

Dupuis, F., M. Lemieux et D. Gosselin, 1992, Conséquences de la sous-spécification des traits de Agr dans l'identification de pro, Language Variation and Change 3, pp. 275-299.

Emonds, J., 1978, The Verbal complex V'-V in French, Linguistic Inquiry 9, pp. 151-175.

Enç, M., 1987, Anchoring Conditions for Tense, Linguistic Inquiry 18, pp. 633-657.

Englebert, A., 1982, L'opposition NE / NE... PAS en ancien français : une question d'incidence? Mémoire de Licence, Université Libre de Bruxelles.

Englebert, A., 1985, L'opposition NE / NE..PAS en ancien français, Revue de Linguistique Romane, 49, pp. 365-378.

Engver, K., 1972, Place de l'adverbe déterminant un infinitif dans la prose du français contemporain, Studia Romanica Upsaliensia 7, Stockholm, Almqvist et Wiksell.

Galet, Y., 1971, L'Evolution de l'ordre des mots dans la phrase française de 1600 à 1700. La Place du pronom personnel complément d'un infinitif régime, Paris, PUF.

Gougenheim, G., 1974, Grammaire de la langue française du seizième siècle, Paris, Picard.

Hirschbühler, P. et M. Labelle, 1992, Changes in Verb Position in French Negative Infinitival Clauses, ms, Université d'Otawa et UQAM, 29 p., Second Diachronic Generative Syntax Workshop, University of Pennsylvania, 6 novembre 1992.

Hirschbühler, P. et M. Labelle, 1993a, Le statut de (ne) pas en français contemporain, Recherches Linguistiques 22, Université de Paris VIII.

Hirschbühler, P. et M. Labelle, 1993b, From ne V pas to ne pas V and the syntax of pas, ms., Université d'Ottawa et UQAM, 29 p.

Kayne, R., 1989, Null Subjects and Clitic Climbing, dans O. Jaeggli et K. Safir (eds.) The Null Subject Parameter, p. 239-262, Dordrecht, Kluwer Academic Publishers.

Kayne, R., 1990, Romance Clitics and PRO, dans J. Carter, R.-M. Déchaine, B. Philip et T. Scherer, Proceedings of NELS 20, vol. 2, pp. 255-302, Amherst, GLSA, University of Massachusetts.

Kayne, R., 1991, Romance Clitics, Verb Movement, and PRO, Linguistic Inquiry 22, pp. 647-686.

Kempchinsky, P., 1992, Clausal Complements and Case Theory in Romance, Probus, pp. 17-32.

Krifka, M., 1991, Some Remarks on Polarity Items, dans D. Zaefferer (ed.), Semantic Universals and Universal Semantics, pp. 150-189, Groningen-Amsterdam Studies in Semantics, Berlin, Foris Publications.

Kroch, A., 1989, Reflexes of Grammar in Patterns of Language Change, Language Variation and Change, 1, pp. 199-244.

Laenzlinger, Ch., 1990, Le déplacement des clitiques et l'analyse DP, mémoire de recherche, Faculté des lettres, Université de Genève.

Lois, X., 1989, Aspects de la syntaxe de l'espagnol et théorie de la grammaire, thèse de doctorat nouveau régime, Paris, Université de Paris VIII. 
Lujan, M., 1980, Clitic Promotion and Mood in Spanish Verbal Complements, Linguistics 18, pp. 381-484.

Martineau, F., 1988, Le placement des pronoms objets dans les constructions infinitives chez Philippe de Vigneulles, Revue québécoise de linguistique théorique et appliquée, 7, pp. 157-173.

Martineau, F., 1990, La montée du clitique en moyen français : une étude de la syntaxe des constructions infinitives, thèse de doctorat, Université d'Ottawa.

Muller, Cl., 1991, La négation en français, Genève, Droz.

Pearce, E., 1990a, Parameters in Old French Syntax. Infinitival Complements. Kluwer. Dordrecht.

Pearce, E., 1990b, An Analysis of Negated Infinitives in Middle French, Wellington Working Papers in Linguistics 2, pp. 31-45.

Pearce, E., 1991, Tense and Negation: Competing Analyses in Middle French, Parasession on Negation, CLS (à paraître en 1993).

Picallo, M.-C., 1990, Modal Verbs in Catalan, Natural Language and Linguistic Theory 8, pp. 285-312.

Pollock, J.-Y., 1989, Verb Movement, Universal Grammar, and the Structure of IP, Linguistic Inquiry 20, pp. 365-424.

Raposo, E., 1987, Case Theory and Infl-to-Comp: The Inflected Infinitive in European Portuguese, Linguistic Inquiry 18, pp. 85-110.

Rivero, M.-L., 1988, The Structure of the Clause and V-movement in the Languages of the Balkan, ms., version révisée à paraître dans Natural Language and Linguistic Theory.

Rivero, M.-L., 1990, The location of Non-Active Voice in Albanian and Modern Greek, Linguistic Inquiry 21, pp. 135-146.

Rizzi, L., 1982, Issues in Italian Syntax, Dordrecht, Foris.

Roberts, I., 1992, Restructuring in Old French, ms., 31 p., Second Diachronic Generative Syntax Workshop, University of Pennsylvania, 6 novembre 1992.

Rochette, A., 1988, Réseau de corrélations : sujet nul, montée et placement des clitiques et le caractère nominal des infinitives, Revue québécoise de linguistique théorique et appliquée 7, Aspects de la syntaxe historique du français, pp. 175-192.

Santorini, B., 1989, The Generalization of the Verb-Second Constraint in the History of Yiddish, thèse de doctorat, Philadelphia, University of Pennsylvania.

Stowell, T., 1982, PRO in Finite Clauses. A Study of the Inflectional Heads of the Balkan Languages, thèse de doctorat, New York, City University of New York.

Vikner, S., 1991, Verb Movement and the Licensing of NP-Positions in the Germanic Languages, 2e version, Ms., 333 p., Université de Stuttgart.

Wackernagel, J., 1892, Über ein Gesetz der indogermanischen Wortstellung. Indogermanische Forschungen 1, pp. 333-436.

\section{Sources et références des exemples}

Alcripe, Philippe d' [c. 1575-1578] La Nouvelle Fabrique des excellents traicts de vérité (Livre pour inciter les resveurs tristes et melancholiques à vivre de plaisir).

Anonyme [c. 1400] Les XV joies de mariage, publ. par Jean Rychner, Droz, Genève, 1957. 
Anonyme [c. 1462] Les Cent Nouvelles Nouvelles, édité par P. Champion, 1828, Droz, Paris. [CNNA]

Anonyme [c. 1450] Les Quinze Joies de mariage, éd. par J. Rychner, Droz, Genève, 1967.

Anonyme [2è moitié du 15è s.] Journal d'un bourgeois de Paris, Le Livre de Poche 4522, Paris.

ARTFL [banque de données du TLF], Department of Romance Languages and Literatures,

University of Chicago. [ARTFL]

Bayle, P. [1686] Comment. Philos. Paroles J-C., ARTFL.

Bergerac, Cyrano de [1655] Voyage dans la lune et Histoire comique des Etats et Empires du soleil, Collection 10/18, Union générale d'Editions. [Voyage]

Boileau-Narcejac [1955] Les Louves. Le Livre de Poche, Paris.

Boileau-Narcejac [1955] Les Diaboliques. Le Livre de Poche, Paris.

Bonaventure des Périers [c. 1540] Nouvelles récréations et joyeux devis, éd. par Kasprzyk, Champion, 1980.

Bossuet, J.-B. [1682] Discours sur l'histoire universelle, Paris, Garnier Flammarion, 1966.

Brantôme [1590] Les Dames Galantes. Ed. Maurice Rat. LeLivre de Poche, Librairie générale française, 1962.

Cazotte, J. [1772] Le diable amoureux, Paris, Garnier Flammarion.

Chasles, R. [1713] Les illustres françoises, ARTFL.

Corneille, P. [1629] Mélite. Théâtre complet. Paris, Classiques Garnier, 1960-1962.

Crébillon, C.Fils. [1736-1738] Les égarements du cœur et de l'esprit.

Crébillon, C.Fils. [1771] Lettres athéniennes, ARTFL.

Daudet, A. [1868] Le petit chose, Le Livre de Poche, Paris.

Des Masures, L. [1566] David fugitif. Ed. par Ch. Compte, Cornely et Cie, 1907.

Diderot, D. [1784]. Jacques le Fataliste, Paris, Le Livre de Poche.

Du Fail, N. [1547] Propos rustiques et Baliverneries, ed. de la Borderie, Slatkine Reprints, Geneva, 1970 (réimpression de l'édition de 1878, Paris).

Dumas, A., père [1844] Les Trois Mousquetaires, Le Livre de Poche, Paris.

Flore, J. [1537] Contes amoureux, ed. Pérouse et al., 1980.

Gide, A. [1925] Les faux-monnayeurs. Paris, Le Livre de Poche.

Habanc, V. [1585] Nouvelles Histoires tant Tragiques que Comiques, éd. par J.-C. Arnould et R.A. Carr, Droz, Genève 1989.

La Boétie, E. de [1546] « Discours de la servitude volontaire », dans CEuvres complètes d'Estienne de la Boétie, ed. par Louis Desgraves, William Blake \& Co., 1991.

La Boétie, E. de [1561] « Mémoire sur la pacification des troubles ", dans CEuvres complètes d'Estienne de la Boétie, éd. par Louis Desgraves, William Blake \& Co., 1991.

Lafayette, Mme de [1678] La Princesse de Clèves, Le livre de Poche, Paris.

Lainé, P. [1984] Trois petits meurtres. Le Livre de Poche, Paris.

La Tour Landry, G. de. [1372] Le Livre pour l'Enseignements de ses Filles, ed. by Anatole de

Montaiglon, Paris, P. Jannet, 1854. 
Leblanc, M. Les aventures d'Arsène Lupin, Collection Bouquins, Paris.

Leroux, G. « Le Mystère de la chambre jaune » \& « Le Parfum de la dame en noir », dans CEuvres complètes, Collection Bouquins, Paris.

Marguerite de Navarre [1549] L'Heptaméron, éd. par Michel François, Garnier, Paris, 1950.

Molière [1671] Le bourgeois gentilhomme. Théâtre complet, T. 4, Paris, Gallimard.

Monluc, Blaise de [1571-1577] Commentaires, éd. par P. Courteault, Bibliothèque de la Pléiade, Gallimard, Paris.

Montaigne. Essais. ed. Pierre Villey et Verdun-L. Saunier, PUF, Paris, 1965 et 1968 (concordance de Leake, Roye E., Genève, Droz, 1981).

Rat, M. [1967] La langue française en Belgique, La chronique du langage, Revue des deux mondes, 1967 :602. [RdDM].

Riccoboni, Mme [1767] Histoire de Miss Jenny. Genève, Droz.

Sand, G. [1847-1848] François le Champi. Le livre de Poche, Paris.

Tallemant des Réaux, G. [1657-1659] Le Cardinal de Richelieu, sa famille, son favori Bois-Robert, Bruxelles, Edition Complexe, 1990.

Troyes, Nicolas de [1536] Le Grand Parangon des Nouvelles Nouvelles, éd. par Kasprzyk, Paris, Didier, 1970.

Vigneulles, Ph. de. [1505-1515] Les Cent Nouvelles Nouvelles, ed. Ch. Levingston, 1972, Droz, Genève. [CNNV]. Concordance lemmatisée de P. Hirschbühler, M.-O. Junker et D. Potvin, Université d'Ottawa, 1988).

Vigneulles, Ph. de. Journal. Gedenkbuch des Metzer Bürgers Philippe von Vigneulles, ed. H. Michelant, Stuttgart, 1852, réédité chez Rodopi, Amsterdam 1968.

\section{NOTES}

1. Evidemment, si l'on adopte une structure du type de (1b), certains aspects du système explicatif décrit ci-dessous sont à revoir.

2. On aurait une confirmation directe de l'hypothèse de Pollock si, en français contemporain, des verbes du type de pleuvoir, qui ne sélectionne aucun argument et donc n'a apparemment aucun rôle thêta à transmettre, pouvaient apparaître à gauche de pas dans les infinitivales. Le fait qu'ils ne le peuvent pas force à dire que, contrairement aux apparences, de tels verbes assignent des rôles thêta ou des quasi-rôles thêta de nature obscure. Pour qui est de l'approche des modaux en termes de rôles thêta adjoints (de type adverbial), le cas du sujet de vouloir est particulièrement problématique : il est difficile d'imaginer quelle sorte de rôle thêta adverbial il pourrait avoir. Ceci n'empêche qu'il est vrai que vouloir (ou ses équivalents dans d'autres langues) peut développer des emplois modaux (il veut pleuvoir) ou s'aligner sur les modaux à certains égards (par exemple, dans les constructions à restructuration de l'italien). Mais il est douteux que lorsque le verbe n'est pas employé comme un modal (...et de ne vouloir pas qu'on lui fasse ... Rousseau, 1776, ARTFL ; ... accusaient ce parti de ne vouloir point d'assemblée nationale ... Las casès, 1823, ARTFL ; ... aussitôt de ne lui vouloir pas de bien. Boylesve, 1902, ARTFL), les propriétés qu'il pourrait partager avec les verbes modaux devraient être interprétées comme indiquant qu'il n'assigne pas de rôle thématique, en particulier à l'argument externe. De plus, comme nous le verrons dans la discussion des données historiques, l'hypothèse de l'opacité de $\mathrm{T}$ [-fini] amène naturellement à se demander quel changement dans les traits de cette catégorie aurait converti 
cet élément initialement «transparent» (permettant la montée du verbe plein) en élément "opaque".

3. Nous nous intéressons ici aux subordonnées infinitives. Comme il nous l'a été rappelé au colloque La Négation, on trouve ne dans les infinitives prohibitives dès l'ancien français. Pearce (1990a :280) suggère que l'absence d'infinitivales négatives gouvernées en ancien français est une indication de ce que, à cette époque, les propositions de ce type étaient des projections de V. L'apparition de la négation ne ou non dans ce contexte en moyen français signalerait un changement dans le statut catégoriel de l'infinitivale, qui serait désormais un IP au minimum.

4. Les exemples sont rares au XVe siècle. Dans son corpus relativement important qui va de La Tour Landry (1372) à Philippe de Vigneulles (1515), Pearce (1991) relève, sur un total de 73 subordonnées à l'infinitif avec ne, 10 exemples de type ne le $V$ pas (tous types de verbes confondus), un de type ne le pas $V$ (provenant de nos données de Vigneulles) et aucun de type ne pasle V.

5. Le clitique seule devant pas ou point est attesté tout au long du XVIIIe siècle pour l'ensemble des clitiques et la construction se perpétue encore durant le XIXe siècle au moins pour en et pour $y$.

6. Pour les modaux nous avons interrogé la totalité des données de ARTFL. Pour les verbes auxiliaires et les verbes pleins, nous avons relevé toutes les occurrences de pas et point pour les périodes suivantes : 16è siècle, 1600-29, 1650-55, 1678-79, 1683-84, 1700-07, 1735-36, 1750-53, 1783-84, 1810-18, 1835-36, 1845-46, 1857-58, 1870-72, 1880-83, 1895-96, 1905-06, 1915-16, 1925, $1935,1940-41,1945,1950-64$. A cela nous avons ajouté les exemples relevés lors de nos propres lectures (cf. Sources et références). Le nombre d'auteurs inclus jusqu'ici dans chaque période est le suivant : 15è. (3), 1500-1549 (8), 1550-1599 (7), 1600-1649 (36), 1650-1699 (20), 1700-1749 (21), 1750-1799 (23), 1800-1849 (28), 1850-1899 (35), 1900-1949 (42), 1950-1984 (21). Etant donné la quantité des données déjà relevées et la distribution claire des faits, nous ne pensons pas que les tendances générales seront différentes pour les périodes couvertes par ARTFL lorsque ce corpus aura été dépouillé entièrement. Nous nous attendons à ce que les choses soient différentes pour les modaux et les auxiliaires pour la deuxième moitié du 20è siècle lorsque suffisamment de données postérieures à 1964, c.à.d. postérieures aux derniers textes de ARTFL, auront été relevées.

7. Un coup d'œil rapide sur les données suggère qu'un recomptage écartant les emplois de vouloir prenant comme complément une phrase ou un NP devrait montrer que la construction moderne du modal à l'infinitif est moins importante aux XVIIIe et XIXe siècles que ne le suggère la table 1.

8. Les auteurs étudiés par Engver sont: Jean Anouilh, Marcel Aymé, Pierre Daninos, Henry de Montherlant, Françoise Sagan, Michel de Saint Pierre, Jean-Paul Sartre, George Simenon ; à cela s'ajoutent 36 numéros du Figaro (7 janvier au 11 mars 1963).

9. En outre, telle que proposée, cette analyse n'est pas conforme à la théorie des barrières, qui ne permet pas qu'une catégorie maximale a, AgrP ici, acquière le statut de barrière par héritage d'une autre catégorie maximale $\mathrm{b}$, VP dans le cas présent, pour un élément $\mathrm{g}$ ( $\mathrm{V}$ ou plus correctement Agr) qui n'est déjà plus contenu dans $\mathrm{b}: \mathrm{V}$ est déjà adjoint à Agr, et AgrP ne peut être barrière par héritage au déplacement de [Agr V-Agr]. De même, AgrP ne peut devenir une barrière par héritage au déplacement des pronoms clitiques, si ce déplacement se fait de tête à tête.

Pour Pollock, la montée du verbe à Agr et à T[-fini] n'est pas bloquée par la présence de barrières, puisque un verbe monté peut L-marquer la catégorie maximale qu'il gouverne, qu'il s'agisse de VP, de AgrP (en l'absence de négation), ou de NegP. Dans les phrases avec négation, l'hypothèse que AgrP n'est pas une barrière inhérente fait qu'il n'y a pas de barrières active entre $\mathrm{T}$ contenant V-Agr et la trace de V-Agr dans Agr; en particulier, ni NegP ni AgrP ne comptent comme barrières. 
10. Martineau (1990) et Pearce (1991) n'en notent aucun dans notre concordance des Cent Nouvelles Nouvelles, mais elles $\mathrm{n}$ 'ont considéré que les exemples avec clitique.

11. Martineau (1990:203-207) suggère que le mouvement de $V$ dans $T$ par-dessus NegP serait facultatif, étant donné que dans de nombreux cas, le pronom faible est séparé du verbe infinitif par des adverbes et des particules négatives. Les données concernant les particules négatives pas et point devraient toutefois être examinées de plus près, notamment en distinguant les exemples introduits par non de ceux introduits par ne. En effet, les exemples introduits par non sont systématiquement du type (clit) non point/pas V. Ceux introduits par ne sont normalement du type $n e$ (clit) $V$ point/pas. Les exemples du type ne (clit) point $V$ sont rarissimes au début du XVIe siècle. Si pas et point sont adjoints à VP, comme nous le suggérons dans le texte, et que le verbe bouge systématiquement au moins jusqu'à la première catégorie fonctionnelle externe au VP (Infn dans l'arbre (13)), on semble devoir dire que plusieurs éléments, comme ceux mentionnés par Martineau (1990, p. 203), dont mieux, plus, jamais, rien, bien, peuvent être placés entre la tête fonctionnelle recevant le clitique (Agr en (13)) et Infn. Une meilleure compréhension des faits requiert une étude plus approfondie des données relatives aux adverbes.

12. Tout comme pour le moyen français, une explication alternative des faits de l'italien peut être envisagée (cf. Hirschbühler et Labelle 1993b).

13. Belletti (1990: 84-85) suggère que le mouvement à une tête fonctionnelle comme Agr en italien est imposé par une propriété morphologique de Agr: si Agr sélectionne morphologiquement V (c.à.d. si Agr est en fait Agr-1), l'amalgame entre V et Agr se fait par mouvement de $\mathrm{V}$; Une telle approche ne semble pas propre à rendre compte de l'évolution du français (cf. Hirschbühler et Labelle 1993b). Il est en effet peu satisfaisant de supposer, pour le XVIIe par exemple, que Agr a deux visages : Agr-1, qui sélectionne aussi bien les $\mathrm{V}$ pleins que les auxiliaires et les modaux, et Agro, qui sélectionne les $\mathrm{V}$ pleins uniquement.

14. Ainsi, l'opinion de Vaugelas (1585-1650) concernant la position de pas et point par rapport au verbe infinitif, citée par Brunot (1966: tome III, deuxième partie, p. 662), pourrait refléter une telle différence stylistique: "pas et point ont beaucoup meilleure grace, estans mis devant qu'apres, par exemple: pour ne pas tomber dans les inconveniens ou pour ne point tomber dans les inconvénients [sic] est bien plus elegant que de dire pour ne tomber pas, ou pour ne tomber point dans les inconvenients» (II, 128-129).

15. Dans notre corpus limité, ne pas $V=10$; ne point $V=2$; ne $V$ pas $=2$; ne $V$ point $=15$.

16. Englebert met également en évidence dans son mémoire de 1982 et dans son article de 1985 des différences de portée de la négation selon que pas/point étaient présents ou non.

17. Muller mentionne aussi comme indiquant l'existence d'un pas/point négatif l'apparition de ces termes sans la présence de ne dans les questions totales ne préjugeant pas d'une réponse négative (cf. Brunot 1967, Tome II : 473 et Gougenheim 1974, p. 218). En fait, la situation est plus complexe. Ainsi, au XVe siècle, époque où on ne note ordinairement pas de négation de phrase sans ne lorsque le verbe est conjugué, il n'est pas difficile de trouver des interrogatives totales sans ne n'invitant pas de réponse négative. C'est également le cas dans la prose classique, qui n'omet normalement pas le ne dans les assertives négatives. Krifka $(1991,154,164)$ prend soin de mentionner que les termes de polarité négative peuvent apparaître dans des questions qui ne suggèrent pas une réponse négative.

18. Bouchard analyse les constructions du type ne être/avoir pas $X$, comme des cas de négation de constituant. Bien qu'il donne certains exemples clairs d'une distinction sémantique selon que l'auxiliaire est à gauche ou à droite du forclusif, nous ne pensons pas que cela suffise à exclure l'analyse en termes de mouvement de verbes pour les auxiliaires en français contemporain. En effet, certains exemples agrammaticaux expliqués par Bouchard sur la base du fait qu'ils ne se prêtent pas à une analyse en termes de négation de constituant étaient courants jusqu'à la fin du XIXe siècle au moins. Bien sûr, la réduction du nombre de cas de montée de l'auxiliaire a pu donner lieu à une grammaire sans montée dans les infinitivales. Mais s'il y a négation de constituant, elle doit être plus restreinte que ce qui est envisagé par 
Bouchard puisque certains des exemples de négation de constituant qu'il donne sont très mauvais, même en contexte contrastif, contrairement à ce qu'il rapporte ('Les enfants n'ont lu pas cette page mais la suivante - Bouchard rapporte un jugement ' ?'). Il est à noter que, si l'exemple suivant de Pollock, N'être pas heureux est une condition pour écrire des romans, repris par Bouchard, était une négation de constituant sans montée de l'auxiliaire, on s'attendrait à ce qu'un exemple parallèle mais avec un verbe plein ait un statut semblable, en particulier dans un contexte contrastif, ce qui n'est pas le cas: *Ne sembler pas heureux est une condition pour écrire des romans. Les exemples avec être et avoir ne semblent pas requérir de contexte contrastif.

19. Cf. Kroch (1990: 222) pour un argument du même type pour have et be en anglais. (Nous n'adoptons pas l'analyse de Pollock, selon laquelle être lexical, c'est être là, et avoir possessif, comme dans Jean a une voiture, c'est plus ou moins Avoir [chez Jean [une voiture [être là]]].)

20. Si les propositions compléments des verbes modaux ne projettent pas jusqu'à $C$, l'ancrage de la subordonnée pourrait se faire directement de Tprincipal à T subordonné.

21. Pour ce qui est de vouloir, rappelons aussi que ce verbe partage des propriétés bien connues avec pouvoir et devoir, en particulier la possibilité de déclencher la restructuration et de permettre la montée longue du clitique : cette propriété peut être une de celles amenant le locuteur à traiter ce verbe de manière parallèle aux verbes pouvoir et devoir, avec pour conséquences la montée de vouloir infinitif à $\mathrm{T}$.

22. Dans les exemples de Montherlant rassemblés par Engver, il y en a 27 où le verbe plein à l'infinitif se trouve à gauche du forclusif. Il vaut la peine de noter que la position postverbale de pas semble être favorisée si l'infinitif est suivi d'un adverbe ou d'un substantif précédé de l'article indéfini ou partitif, éléments qui se combinent facilement avec pas (Engver, p. 20). L'exemple qui suit, contrairement à celui déjà donné, est de ce type: "Ainsi on en attaque certains de ne souffrir pas assez, quand on ferait mieux de... » (Montherlant, Le Solstice de Juin, p. 145, dans Engver, p. 20).

\section{RÉSUMÉS}

Nous examinons l'évolution de la position des morphèmes négatifs pas et point par rapport au verbe infinitif. On observe que le changement de la construction ne Vinf pas/point à ne pas/ point Vinf se fait à des époques différentes selon que le verbe infinitif est un verbe plein, un modal (pouvoir, vouloir, devoir) ou un auxiliaire. Contrairement à la plupart des analyses, nous explorons l'hypothèse que le changement initial consiste en un repositionnement du morphème négatif plutôt qu'en un repositionnement du verbe. Ce changement n'a de conséquence visible que pour les verbes pleins, ceux-ci occupant déjà en moyen français une position structurale moins élevée que celle des auxiliaires et des modaux. Ultérieurement, les modaux adoptent la même position structurale fonctionnelle que les verbes pleins, avec pour conséquence un changement dans la position relative de ces verbes par rapport au morphème négatif. Les auxiliaires alignent graduellement leur comportement sur celui des autres verbes. Nous proposons une analyse du comportement historique distinct de chacune des classes de verbes considérées qui repose sur les propriétés lexicales de ces verbes et leur affinité différente avec la catégorie abstraite Temps.

We study the evolution of the position of the negative morphemes pas and point with respect to the verb in the infinitive. It is shown that the change from the construction ne Vinf pas/point to 
ne pas/point Vinf takes place at different times according to whether the verb is a full (lexical) verb, a modal (pouvoir, vouloir, devoir) or an auxiliary. Contrary to most current analyses, we explore the idea that the initial change consists in a repositioning of the negative morpheme rather than of the verb. This change manifests itself overtly only in the case of lexical verbs, these occurring, already in Middle French, in a position structurally lower than that of auxiliaries and modals. Later, modals come to occupy the same structural position as full verbs, with a resulting change in the respective position of modals and the negative morphemes. Auxiliaries progressively align their behavior on that of the other verbs. Our analysis of the historically different evolution of each type of verb is based on their lexical properties and their distinct affinities with abstract Tense.

\section{AUTEURS}

\section{PAUL HIRSCHBÜHLER}

Université d'Ottawa, phhaf@acadvm1.uottawa.ca

\section{MARIE LABELLE}

Université du Québec à Montréal, Labelle.Marie@uqam.ca 